\title{
Apropiación humana de la producción primaria en el Chaco Seco
}

\author{
Carla V. Rueda ${ }^{1,2 \bowtie}$; Germán Baldi ${ }^{1}$; SAntiago R. Verón ${ }^{3} \&$ Esteban G. Jobbágy ${ }^{1}$ \\ 1. Grupo de Estudios Ambientales - IMASL. Universidad Nacional de San Luis - CONICET, San Luis, Argentina. 2. Facultad \\ de Ciencias Forestales, Universidad Nacional de Santiago del Estero, Santiago del Estero, Argentina. 3. Instituto de Clima y \\ Agua - INTA, Castelar, Argentina.
}

\begin{abstract}
Resumen. El Chaco Seco experimenta altas tasas de deforestación y transformación productiva. Estimamos la magnitud de la apropiación humana de la productividad primaria neta (PPN) aérea de esta región por vías directas (producción ganadera, forestal y agrícola) e indirectas (fuego), su distribución regional, su relación con variables climáticas y socioeconómicas y sus contrastes entre vegetación natural, pasturas y cultivos. Para 69 departamentos se utilizaron datos censales, climáticos y satelitales (período 2002-2006) a partir de los que se estimó la PPN aérea con modelos climáticos, la apropiación de forraje por ganadería con datos de stock animal y modelos metabólicos, la apropiación de productos agrícolas y forestales con estadísticas nacionales y apropiación indirecta de biomasa por fuego a partir sensores remotos. La ganadería, desarrollada bajo vegetación natural y pasturas (90.9\% del área), involucró una apropiación de $248 \mathrm{kgMS} \mathrm{ha}^{-1} \cdot \mathrm{año}^{-1}$, y aumentó en departamentos con mayor fracción de pasturas, ganado agrupado en rodeos más grandes y menores niveles de pobreza. La apropiación forestal, desarrollada bajo vegetación natural (88.7\%), alcanzó $64 \mathrm{kgMS} \mathrm{ha}^{-1}$. $\mathrm{año}^{-1} \mathrm{representados}$ predominantemente por carbón vegetal y leña, y fue mayor en departamentos de clima más húmedo. En la fracción agrícola del territorio (6.3\%), la apropiación directa trepó a $2293 \mathrm{kgMS}_{\text {.ha }}{ }^{-1}$.año ${ }^{-1}$, representada principalmente por grano de soja y maíz. La apropiación indirecta por fuego promedió $746 \mathrm{kgMS} \mathrm{ha}^{-1}$.año ${ }^{-1}$ para todo el territorio. Este flujo estuvo positivamente asociado a la tasa de desmonte, ascendiendo de $\sim 450$ a $>2000 \mathrm{kgMS} \cdot \mathrm{ha}^{-1} \cdot \mathrm{año}^{-1}$ al pasar de departamentos con mínimo a máximo desmonte. En la actualidad, la baja apropiación de la PPN de la vegetación natural del Chaco Seco constituye un incentivo para su reemplazo por pasturas y cultivos anuales. Una actividad ganadera y/o forestal más eficiente en los bosques representa un desafío tecnológico y político prioritario capaz de reconciliar la producción y la conservación en esta región.
\end{abstract}

[Palabras clave: bosque, desmonte, fuego, ganadería, pasturas, productividad primaria neta, uso forestal]

\begin{abstract}
AвSTRACт. Human appropriation of primary production in the Dry Chaco: The Dry Chaco undergoes high rates of deforestation and productive transformation. Here we estimate human appropriation of net aboveground primary productivity (NPP) for this region, both through direct (livestock, logging, and agricultural production) and indirect pathways (burning), and present their regional distributions and their association with climatic and socioeconomic variables and their contrast between natural vegetation, pastures and crops. For all the 69 counties encompassed by the region we collected productive and climatic statistics and remote sensing data (2002-2006 period). We estimated aboveground NPP based on existing climatic models, forage appropriation based on livestock statistics and metabolic models, forest and agricultural product appropriation using national statistics, and indirect appropriation or consumption by fires based on remote sensing information. Livestock production, based on natural vegetation and pastures (90.9\% of the area) appropriated $248 \mathrm{kgDM} \cdot \mathrm{ha}^{-1} \cdot \mathrm{yr}^{-}$ 1 , increasing in counties with larger pasture proportion, cattle grouped in large herds, and lower poverty levels. The appropriation of forest products, restricted natural vegetation (88.7\% of the area), reached 64 $\mathrm{kgDM} \cdot \mathrm{ha}^{-1} \cdot \mathrm{yr}^{-1}$, represented predominantly by charcoal and firewood, increasing in more humid counties. In the agricultural fraction of the territory (6.3\% of the area), direct appropriation reached $2293 \mathrm{kgDM} \cdot \mathrm{ha}^{-1} \cdot \mathrm{yr}^{-1}$, represented mainly by soy and corn grain. The indirect appropriation of NPP through fires averaged 746 $\mathrm{kgDM} . \mathrm{ha}^{-1} \cdot \mathrm{yr}^{-1}$ for the whole territory. This flux was positively associated to the rate of deforestation, climbing from $\sim 450$ to $>2000 \mathrm{kgDM} \cdot \mathrm{ha}^{-1}$. $\mathrm{yr}^{-1}$ from counties with minimum to maximum deforestation rates. Currently, the low direct appropriation of NPP under natural vegetation in the Dry Chaco involves a strong incentive for its replacement with pastures and annual crops. More efficient livestock and forestry activities in these forest ecosystems represent a technological challenge and a policy making priority capable of reconciling production and conservation in the region.
\end{abstract}

[Keywords: fire, forest, land clearning, livestock, logging, net primary productivity, pasture]

\section{INTRODUCCIÓN}

La productividad primaria neta (PPN) representa la transformación de energía solar en material vegetal y es el proceso que sostiene el flujo energético en los ecosistemas y el soporte de múltiples necesidades humanas

$\triangle$ Grupo de Estudios Ambientales - IMASL. Universidad Nacional de San Luis - CONICET, San Luis, Argentina. carlavrueda@gmail.com
(Schlesinger 1997; Jorgensen 2002; Odum 2002). El aprovechamiento humano de los ecosistemas terrestres involucra la captura de una fracción de la PPN en forma de productos cosechados. La intensidad de esta cosecha depende del tipo de uso (e.g. pastoreo de vegetación natural, cultivo de pasturas,

Editora Asociada: Ana Cingolani

Recibido: 8 de agosto de 2012; Fin de arbitraje: 22 de septiembre; Ultima versión: 12 de febrero; Aceptado: 17 de febrero. 
cultivos agrícolas anuales), de las tecnologías aplicadas y, en sentido más amplio, del contexto socio-económico y cultural de los sistemas productivos (Martínez Alier 1998; Haberl et al. 2002, 2004; Baldi \& Jobbágy 2012).

La apropiación humana de la PPN comprende vías directas e indirectas (Haberl et al. 2004; Krausmann et al. 2007; Erb et al. 2009). La apropiación directa involucra la cosecha de productos vegetales (e.g. granos, madera, fibras) y animales (e.g. carne, leche, lana), considerando en el caso de los primeros la tasa de extracción de biomasa vegetal y en el de los segundos el consumo de biomasa vegetal que permitió generarlos (Haberl et al. 2001). La apropiación indirecta de la PPN incluye vías disipativas e inhibitorias. La primera incluye disturbios antrópicos como la quema o la remoción mecánica de biomasa (e.g. rolado). La segunda comprende la reducción de la PPN inicial de un ecosistema como resultado de las transformaciones que lo ponen en producción (e.g. reemplazo de bosques por cultivos anuales). Por otro lado algunas transformaciones pueden incrementar la PPN inicial (estimulación) generando una apropiación negativa (e.g. aplicación de riego o plantación de forestaciones en pastizales) (Haberl et al. 2004; Krausmann et al. 2007; Erb et al. 2009). Cabe destacar que la PPN de un ecosistema es sólo una dimensión de los múltiples servicios que éstos prestan a la sociedad (e.g. provisión de agua y hábitat, regulación climática) y que las intervenciones humanas, aun cuando no generen una apropiación neta de su productividad primaria, pueden afectar la provisión de estos otros servicios (Laterra et al. 2012).

La fracción de la PPN terrestre apropiada por los humanos se ha incrementado a lo largo de la historia, impulsada por el crecimiento de la población y de su consumo y favorecida por los cambios tecnológicos. Según Haberl (2001), se pasó de niveles cercanos al 5\% en sociedades agrícolas pre-industriales a $30 \%$ en sociedades industriales, y se proyecta llegar al $50 \%$ a mediados de este siglo. Otras estimaciones sugieren valores actuales que van del 20 al 32\% de apropiación de la PPN terrestre global (Vitousek et al. 1986; Rojstaczer et al. 2001; Imhoff et al. 2004; Haberl et al. 2007). En áreas originalmente ocupadas por bosques secos subtropicales, el uso de la tierra predominante corresponde a la ganadería y la explotación forestal de la vegetación natural, $\mathrm{y}$ en mucho menor medida a su reemplazo por cultivos, que pueden ser pastoriles o agrícolas (Baldi \& Jobbágy 2012). Este es el caso del Chaco, el Brigalow en Australia, los Mesquitales en Norteamérica, y el MiomboMopane en África, en donde actualmente el área cultivada no supera el 16\% (Tinkler 2004; Chidumayo \& Gumbo 2010; Seabrook et al. 2006; Baldi \& Jobbágy 2012). Una excepción notable es el noroeste de India y noreste de Pakistán, en donde tierras que comparten las condiciones climáticas de las regiones anteriores y fueron ocupadas originalmente por bosques secos, son hoy cultivadas en un 75\% (Baldi \& Jobbágy, 2012).

La apropiación de la PPN por la vía ganadera en bosques secos suele verse limitada por dificultades en el aprovechamiento de la vegetación natural por el ganado por baja accesibilidad (Kunst et al. 2006) o calidad del forraje (Garbulsky et al. 2005), por el difícil acceso al agua de bebida animal, o por condiciones de infraestructura y limitaciones socioeconómicas (Miñon et al. 1991; Quiroga et al. 2009). Comparadas con regiones más húmedas, las tierras secas por lo general presentan proporcionalmente una menor carga animal y producción ganadera respecto a su PPN aérea (Oesterheld et al. 1992), demostrando que limitaciones ecológicas y/o humanas restringen su apropiación (Oesterheld et al. 1998; Blanco et al. 2008). Asimismo en estos sistemas se suele generar una fuerte apropiación disipativa de la PPN por la aplicación prescripta o accidental de disturbios antrópicos como el fuego o la remoción mecánica de biomasa (e.g. rolado) que en algunas casos buscan facilitar la cosecha y calidad de la PPN después del disturbio (Carranza et al. 2000, 2005; Anriquez et al. 2005; Kunst et al. 2006). Finalmente, las intervenciones ganaderas más drásticas que involucran el reemplazo total de la vegetación natural por pasturas o cultivos anuales forrajeros, obtienen una mayor fracción cosechable de la PPN, si bien en muchos casos reducen la PPN alcanzada respecto a la del sistema natural; es decir, estos sistemas elevan la apropiación directa de la PPN acompañándola con una apropiación inhibitoria adicional.

La apropiación de la PPN por la vía forestal en bosques secos se ha centrado históricamente en la provisión de leña y carbón y, en menor medida, de madera. La alta densidad y lignificación sumadas al bajo contenido de humedad del leño de las especies de estos bosques resultan óptimas para la producción de carbón vegetal (FAO 1983; MA 2002; Chidumayo \& Gumbo 2010). Sin 
embargo, la producción de madera aserrada se ve restringida por la predominancia de fustes cortos y tortuosos. En los bosques secos una fracción apreciable de la PPN puede ser apropiada en forma indirecta y disipativa por el fuego (Bond \& Keeley 2005). La frecuencia e intensidad de incendios de la vegetación natural suele ser máxima bajo condiciones intermedias de balance hídrico (i.e. semiaridez), dado que las condiciones más húmedas inhiben la combustión, mientras que las más secas limitan la cantidad de biomasa disponible (Di Bella et al. 2006). Superpuesto a este tipo de controles climáticos, la expansión de cultivos y pasturas suele ser precedida por fuegos que eliminan la biomasa del bosque (Kunst et al. 2003; Garbulsky et al. 2005; Bravo et al. 2010; Verón et al. 2012), si bien en sistemas secos una vez establecidos los cultivos la actividad de incendios tiende a reducirse (Di Bella et al. 2006).

Conocer cuáles son las tasas de apropiación de la PPN en una región y cómo varía geográficamente en respuesta a controles naturales y humanos nos permite diagnosticar la eficiencia productiva de sus usos, reconocer oportunidades para mejorarlos, e identificar compromisos con otras funciones de la PPN, tales como el soporte de vida silvestre o el almacenamiento de carbono. En el Chaco Seco Argentino, este desafío ha recibido poca atención y cobra urgencia ante los veloces cambios del uso de la tierra de esta región. Los objetivos de este trabajo son (i) estimar la magnitud de la apropiación humana de la PPN por vías directas (producción ganadera, forestal y agrícola) e indirectas disipativas (fuego) e inhibitorias (reducción de la PPN asociada a las intervenciones productivas) en el Chaco Seco; (ii) caracterizar la distribución espacial de la apropiación humana de la PPN y su relación con variables climáticas y sociales y (iii) comparar su magnitud bajo vegetación natural, pasturas implantadas y cultivos anuales. Para ello se utiliza información proveniente de estadísticas socioeconómicas, ganaderas, agrícolas y forestales, información satelital e información de campo disponible para la región.

\section{MATERIALES y MÉTOdOS}

\section{Área de estudio}

Este trabajo se centra en el Chaco Seco Argentino (Brown et al. 2006). La precipitación media anual en esta región varía entre 350 y 700 mm y la vegetación original comprende bosques xerofíticos y arbustales incluyendo también sabanas y pastizales (Bucher 1982). La historia de uso posterior a la colonización incluye (i) la ocupación por grandes haciendas ganaderas a partir del siglo XVIII (Navarrete et al. 2005), (ii) la explotación forestal selectiva a principios del siglo XX (Van Dam 1999), (iii) la intensificación ganadera a partir de 1990 (Van Dam 1999; Paruelo et al. 2006), y (iv) el creciente uso agrícola de la región durante las tres últimas décadas (Paruelo et al. 2006). El área de estudio abarca $480000 \mathrm{~km}^{2}$ en el centro norte de Argentina y está integrada por parte de las provincias de Salta, Formosa, Chaco, Córdoba, Catamarca, La Rioja, San Luis y la totalidad de Santiago del Estero, incluyendo 69 departamentos (Figura 2a). Estas entidades administrativas se toman como unidad de análisis. Sólo para un subconjunto de 29 departamentos cuyas condiciones algo más húmedas que el promedio permiten la coexistencia de vegetación natural, pasturas implantadas $(>2 \%$ del área) y agricultura de secano (>6\% del área) se comparó la apropiación directa e inhibitoria de la PPN para los distintos tipos de cobertura. En este análisis se descartaron los departamentos con mayor aplicación de riego a los cultivos (>12\% del área cultivada) para descartar las distorsiones que este aporte de agua puede tener sobre las estimaciones de la PPN y su apropiación en las condiciones de secano, que son el foco de este estudio.

\section{Indicadores de apropiación}

A nivel departamental se estimó la magnitud de la apropiación directa de la PPN por ganadería, explotación forestal y agricultura, y la apropiación indirecta por fuegos aplicando valores de materia seca por unidad de tiempo (año) y superficie (hectárea). La fracción de la superficie del Chaco Seco utilizada para este cálculo varió según el tipo de apropiación estudiado y el tipo de cobertura en el cual tiene lugar. Sobre la base de tres grandes tipos de cobertura (i.e. vegetación natural, pasturas implantadas y cultivos anuales), se adjudicó la producción ganadera al conjunto de vegetación natural y pasturas, la producción forestal exclusivamente a la vegetación natural, y la producción agrícola exclusivamente al área ocupada por cultivos anuales. Si bien pueden existir casos que escapen a estas asignaciones como el consumo de rastrojos agrícolas por el ganado o la cosecha de productos forestales en tierras agrícolas (plantaciones), se consideró a los mismos despreciables. En el caso del consumo de biomasa por fuegos se adjudicó la misma a la totalidad del territorio, incluyendo las tres coberturas mencionadas. Para la apropiación directa ganadera e indirecta por fuegos fue posible discriminar tentativamente la contribución relativa de distintos tipos de cobertura, como se detalla más abajo. Los valores de superficie de las distintas coberturas para cada departamento se obtuvieron de censos nacionales (INDEC 2002, SIIA 2012).

Para calcular la tasa anual de consumo de biomasa del ganado (apropiación directa ganadera) se utilizaron los datos de existencias ganaderas a nivel departamental (INDEC 2002) combinados 
con modelos de requerimientos energéticos para bovinos y caprinos (Información Suplementaria, Anexo 1). Los supuestos más importantes de esta estimación de detallan en el Anexo 1 y se refieren a (i) el peso medio y las ganancias de peso de las distintas categorías animales, (ii) la relación entre la composición de categorías y las tasas reproductivas, y (iii) la digestibilidad media del forraje, considerada del 55\% (Kunst et al. 1999, 2003; Garbulsky et al. 2005). La energía total consumida por el ganado se discriminó en energía de producción y de mantenimiento sobre la base de la composición por categorías de las poblaciones bovinas y caprinas (Anexo 1). Se descartaron otras especies de animales domésticos por ser muy minoritaria su contribución al stock total $(<1 \%)$.

La apropiación forestal consideró la biomasa leñosa cosechada como madera o combustible. Para ello se utilizaron las estadísticas forestales nacionales (período 2002-2006, Dirección de Bosques de la Nación 2006) y estimaciones de consumo doméstico de leña que (Anexo 1). La apropiación de la PPN por fuegos se estimó a partir de un análisis global de Verón et al. (2012), que reporta el promedio de la energía disipada por fuegos durante el periodo 2003-2006, utilizando los productos satelitales MOD y MYD 14CMG colección 4 (Giglio et al. 2006). Superponiendo el mapa departamental a esta información se estimó el promedio anual de energía disipada por fuegos para cada departamento (Anexo 1).

La apropiación de la PPN por agricultura se estimó tomando directamente el valor de rendimientos promedio (i.e. grano cosechado) para las campañas 2002-2006 para 8 cultivos que representan el 99\% del área y 96\% de la producción (soja, trigo, maíz, sorgo, girasol, algodón, poroto seco y cártamo) (SIIA 2012). Se consideró la superficie sembrada de cada cultivo para ponderar su contribución relativa al total de la producción agrícola y la suma de estas superficies determinó el área total agrícola usada para el cálculo final de apropiación de la PPN.

Tanto para estimar la apropiación inhibitoria de la PPN, como para obtener valores relativos de apropiación directa de la PPN fue necesario estimar la PPN aérea media por departamento de cada tipo de cobertura. En el caso de la vegetación natural se usó un modelo que estima PPN en sistemas leñosos a partir de datos de temperatura y precipitación media (Del Grosso et al. 2008). Los datos climáticos fueron obtenidos para los distintos departamentos de la región de estudio de la base "Ten minute climatology data base" (CRU - UEA, New et al.2002). En el caso de las pasturas implantadas, la estimación de PPN aérea se basó en mediciones existentes de PPN aérea de pasturas típicas de la región obtenidas con mediciones de campo (un total de ocho estudios, Cornacchione 2009, Kunst et al. 2006). La PPN área de cultivos agrícolas se basó en dividir los datos de rendimiento por un índice de cosecha estimado (relación grano /biomasa) de 0.45 (Satorre et al. 2004) y aplicarlos al área sembrada anual. El análisis comparativo de la PPN aérea y su apropiación por cosecha bajo vegetación natural, pasturas y agricultura se focalizó en los 29 departamentos que comparten estas coberturas. A partir de estas comparaciones se estimó la apropiación inhibitoria de la PPN aérea.

Con el fin de ilustrar la heterogeneidad de la apropiación de la PPN aérea dentro del Chaco Seco, se seleccionaron como indicadores (i) la apropiación ganadera total $\left(\mathrm{kgMS} / \mathrm{ha}^{-1} \cdot \mathrm{anno}^{-1}\right)$, asî como la contribución relativa que hacen a ésta (ii) los caprinos y (iii) el consumo total que se destina a producción secundaria (complemento de lo destinado a mantenimiento), la apropiación (iv) por cosecha forestal y (v) por fuegos.

\section{Variables climáticas y socioeconómicas}

Se seleccionaron variables climáticas y socioeconómicas para analizar su asociación espacial con los patrones interdepartamentales de apropiación de la PPN dentro del Chaco Seco. Estas variables incluyeron la precipitación media anual (PPT), obtenida de la base de datos arriba descripta y la latitud media (Lat) de cada departamento. Como variables socioeconómicas se consideró a la densidad de población total (rural y urbana, Dens), la fracción de la población rural (\%PR), y la fracción de la población con necesidades básicas insatisfechas (\%NBI), obtenidas a nivel departamental del Censo Nacional de Personas y Hogares (INDEC 2001). Como variables descriptivas de los sistemas de producción ganadera se consideró la fracción de la población animal total de los departamentos (sobre cantidad total de animales) perteneciente a explotaciones de menos de 200 cabezas ( $\mathrm{R}$ chicos), o >1000 cabezas ( $\mathrm{R}$ grandes) en el caso de bovinos, y hatos $<100$ cabezas (H chicos), o >200 cabezas (H grandes) en el caso de caprinos. Dicha información se obtuvo del Censo Nacional Agropecuario (INDEC 2002) y se refiere a explotaciones agropecuarias con límite definido. También se consideró el porcentaje del territorio ocupado por pasturas (pasturas), cultivos anuales (agricultura), y la tasa media de desmonte anual (tasa desmonte); ésta última variable se refirió solamente a los 27 departamentos de Santiago del Estero y consideró el promedio del periodo 20022006 según las estimaciones de Mariot et al. (2007).

Las asociaciones entre indicadores de apropiación y variables climáticas y socioeconómicas se evaluaron mediante el coeficiente de correlación de Pearson y para las asociaciones más relevantes se evaluaron modelos alternativos de ajuste lineal y exponencial (INFOSTAT, Di Rienzo et al. 2009) seleccionados por el criterio de Akaike (1974).

\section{Resultados}

La principal vía directa de apropiación de la PPN aérea de la vegetación natural del Chaco Seco estuvo asociada a la producción ganadera (Figura 1). Esta actividad se caracterizó por ser predominantemente bovina y por presentar un alto costo en 
términos de la fracción de lo consumido que se destina a mantenimiento. El área ganadera de la región, que incluye vegetación natural y pasturas implantadas (90.9\% del territorio), alberga aproximadamente 4 y 1.5 millones de individuos vacunos y caprinos que reparten el consumo medio de $248 \mathrm{kgMS}$.ha- ${ }^{-1}$. ño $^{-1}$ en 98 vs. $2 \%$, respectivamente (Figura 1). Para bovinos el porcentaje promedio de madres sin cría fue de $45 \%$, lo que resultó en un alto desvío de energía a mantenimiento vs. producción (81\% en promedio, Figura 1). Para caprinos, el porcentaje de madres sin cría fue del 52\% y la asignación a producción fue algo más elevada (29\% para toda la región, Figura 1).

La importancia relativa de las distintas vías de apropiación resultó variable a través de la región (Figura 2). La apropiación ganadera de la PPN aérea mostró sus valores máximos (>1000 kgMS.ha-1.año-1) al sudeste de la región (Figura 2b). Los valores más bajos (<150 kgMS.ha- ${ }^{-1}$. año $\left.{ }^{-1}\right)$ se encontraron dispersos en la región, incluyendo los llanos riojanos, este de Salta, y centro de Santiago del Estero. La apropiación ganadera se incrementó con el aumento del área destinada a pasturas (Figura 3). Esta relación indica una apropiación mínima donde no hay pasturas (ordenada al origen de $184 \mathrm{kgMS} \mathrm{ha}^{-1}$. año $^{-1}$ ) y un crecimiento lineal cuando éstas reemplazan la vegetación natural (pendiente, $43 \mathrm{kgMS} \cdot \mathrm{ha}^{-1}$. año ${ }^{-1}$ por cada $+1 \%$ del área bajo pastura). Este análisis sugiere que en cada unidad de superficie de pastura (100\% de pastura en la función descripta) la ganadería se apropiaría de $\sim 4500$ kgMS.ha-
${ }^{1}$.año ${ }^{-1}$ o 24 veces más de lo que hace que bajo vegetación natural. La eliminación del departamento con más alto nivel de pasturas en este análisis no afectó la tendencia general, resultando en un ajuste algo menor $\left(R^{2}=0.61\right.$ vs. 074) pero similar ordenada al origen (187 kgMS.ha- ${ }^{-1}$ año ${ }^{-1}$ ) y pendiente (44.9 kgMS.ha${ }^{1}$. año ${ }^{-1}$ por cada $+1 \%$ ).

Si bien la producción caprina tuvo una contribución regional minoritaria $(2 \%$ de la apropiación ganadera), fue localmente importante en los departamentos del centro de Santiago del Estero y en Ancasti en Catamarca (8 al 18\%, Figura 2e). La fracción de la apropiación ganadera de la PPN aérea que se destinó a producción secundaria alcanzó un promedio para toda la región de 19\%, siendo máxima en Catamarca (Capayán y Santa Rosa, $>26 \%$ ), en donde el porcentaje de madres vacunas con cría es de los más altos de la región, y mínima en Córdoba (Cruz del Eje y Punilla, $<10 \%$ ), en donde sóloel 19\% delas madres vacunas tienen crías (Figura 2f). La apropiación ganadera en los diferentes departamentos además de aumentar con la proporción de superficie bajo pasturas (Figura 3, Tabla 1) se asoció negativamente con la pobreza $(\% \mathrm{NBI})$ y con la predominancia de rodeos vacunos de $<200$ cabezas ( $R$ chicos), y creció hacia el norte(Lat) y con una mayor proporción de hatos caprinos $<100$ cabezas (H chicos) (Tabla 1 ).

La apropiación forestal dela PPN corresponde a las áreas ocupadas por vegetación natural $(88.7 \%)$ dada la ínfima superficie $(<1 \%)$ de cultivos forestales en la región. En promedio

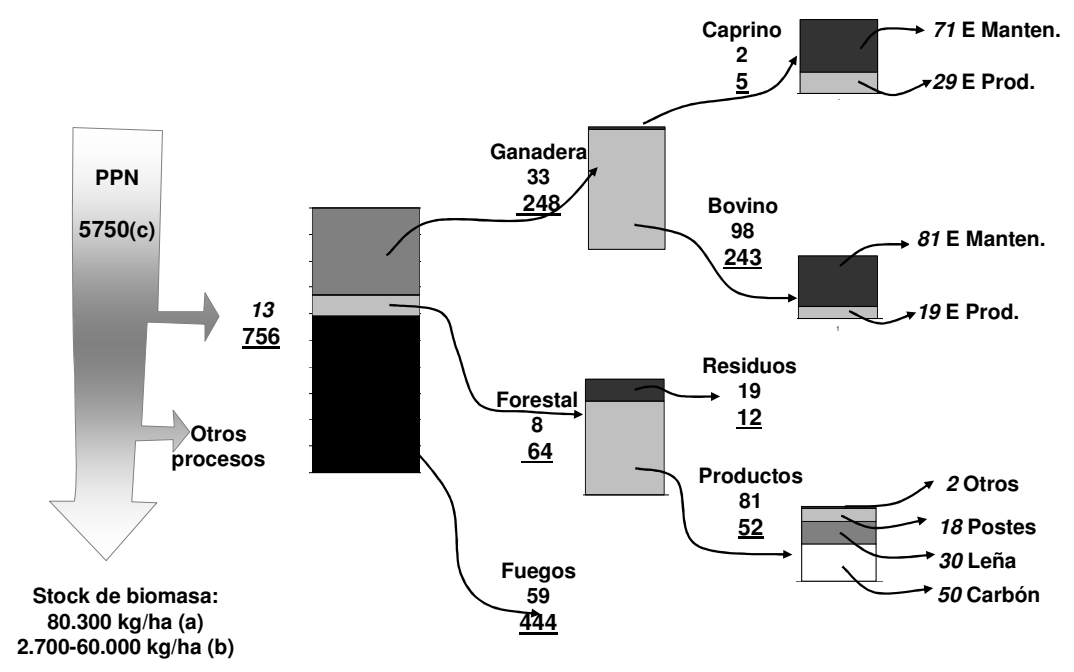

Figura 1. Flujo de apropiación humana de la producción primaria neta (PPN) aérea para la vegetación natural y pasturas implantadas del Chaco Seco. Los valores subrayados indican el flujo anual absoluto de biomasa por unidad de superficie (kgMS.ha- $\left.{ }^{-1} \cdot \mathrm{año}^{-1}\right)$, los valores ubicados por encima de éstos representan porcentajes de partición en cada nivel. La apropiación por fuegos descarta el aporte de las quemas de desmonte y representa una estimación de la disipación media observada en todo el territorio cuando no hay cambios de cobertura (ver Figura 3). Se presentan las existencias de biomasa aérea estimada por (a) Gasparri et al. 2008 y (b) Bonino 2006 y (c) la estimación de PPN aérea media para la región según el modelo de Del Grosso et al. (2008). Otros procesos incluyen herbivoría silvestre y descomposición.

Figure 1. Flow of human appropriation of aerial net primary production for the territory occupied by natural vegetation and pastures in the Dry Chaco. See details in legend in Spanish. 


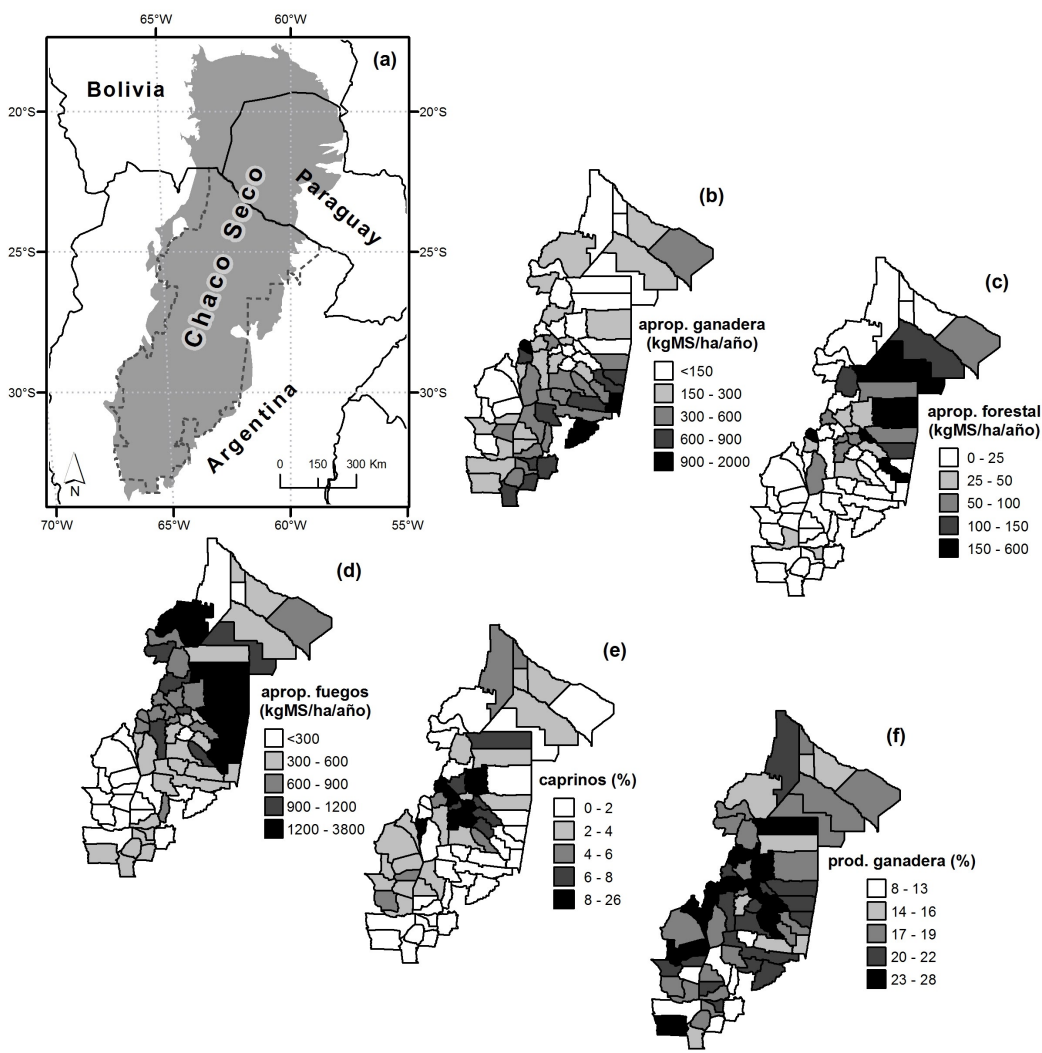

Figura 2. (a) Localización de la ecoregión del Chaco Seco según Olson et al. (2001) y del área de estudio representando 69 departamentos (línea punteada). Apropiación humana de la productividad primaria neta (kgMS.ha ${ }^{1}$.año ${ }^{-1}$ ) por las actividades (b) ganadera, y (c) forestal, y por (d) fuegos. En porcentajes se indica la fracción del consumo ganadero destinado a (e) caprinos y (f) producción (vs. mantenimiento). Los valores de apropiación ganadera se refieren al área de pasturas y vegetación natural y los de apropiación forestal solamente al área de vegetación natural.

Figure 2. Location of the Dry Chaco ecoregion according to Olson et al. (2001) and the study area represented by 69 counties (dotted lines) (a). Human appropriation of net primary productivity $\left(\mathrm{kgDM} \cdot \mathrm{ha}^{-1} \cdot \mathrm{yr}^{-1}\right)$ for (b) livestock consumption and (c) forest product harvesting and (d) and fires. Percentages showing the fraction of allocated to (e) goats and (f) production (vs. maintenance) are shown. See details in legend in Spanish.

total livestock consumption

esta vía de apropiación representó 66 kgMS.ha${ }^{1}$.año ${ }^{-1}\left(64 \mathrm{kgMS}\right.$ ha $^{-1}$.año ${ }^{-1}$ si se aplica al área vegetación natural y pasturas en conjunto, Figura 1). Un $80 \%$ de la producción forestal correspondió a usos energéticos (carbón vegetal y leña). El consumo de esta energía se repartió en forma pareja entre la cosecha declarada, típicamente asociada al uso fuera de la región (52\%), y la que se estimó para el abastecimiento doméstico local (48\%). El NE

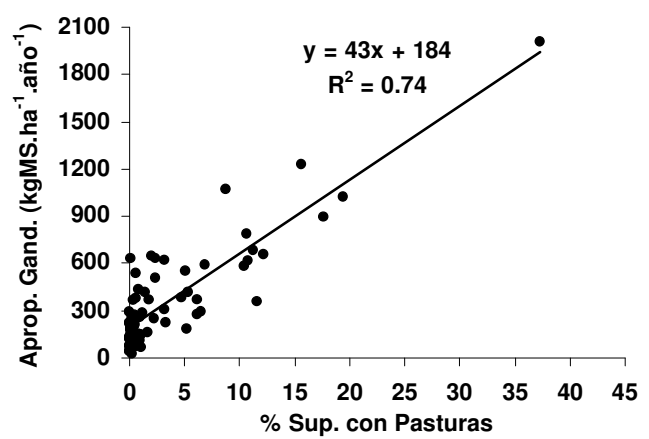

Figura 3. Relación entre la tasa anual de apropiación ganadera de la PPN aérea (vacuno y caprinos) y el porcentaje del área cubierta por pasturas para todos los departamentos del área de estudio.

Figure 3. Relationship between annual rate of appropriation of aboveground NPP by livestock (cattle and goats) and the percentage of the area covered by pastures for all counties in the study area. de la región (Santiago del Estero y Chaco) representa el área de máxima apropiación forestal de la PPN aérea, alcanzando valores de 610 $\mathrm{kgMS} \cdot \mathrm{ha}^{-1} \cdot \mathrm{año}^{-1}$. El uso de madera para aserrado es máxima en el departamento Almirante Brown (Chaco, Figura 2c), donde alcanza 204 kgMS.ha${ }^{1}$.año ${ }^{-1}$. La apropiación forestal estuvo asociada en forma significativa únicamente a la precipitación, siendo mayor en zonas más húmedas, mientras que mostró sólo asociación positiva con la tasa de desmonte fue solo marginal (sólo evaluada para Santiago del Estero).

Si bien la agricultura aún ocupa una fracción minoritaria del Chaco Seco (6.3\%), genera un apropiación directa de la PPN superior a la de la actividad forestal, alcanzando 144 kgMS.ha${ }^{1}$.año ${ }^{-1}$ para la totalidad del territorio y 2293 $\mathrm{kgMS} \cdot \mathrm{ha}^{-1} \cdot \mathrm{año}^{-1}$ si se adjudica la producción sólo al área sembrada con estos cultivos anuales. Sólo en algunos departamentos del este de Santiago del Estero, este de Salta y norte de Córdoba la agricultura cubre más del 15\% del área, destacándose Río Primero, en esta última zona, con $72 \%$ del área bajo agricultura (Información Suplementaria, Figura A2). Es en esta zona también donde se registran las apropiaciones directas más altas bajo agricultura, superando los 2500 kgMS.ha ${ }^{-1} \cdot a^{0} \mathrm{O}^{-1}$. 
Tabla 1. Coeficientes de correlación y valores de significancia para la asociación entre indicadores de apropiación de la PPN y variables climáticas, socioeconómicas y productivas. Se incluyen variables geográficas como precipitación media anual (PPT) y latitud (Lat), demográficas y sociales como densidad de población (Dens), proporción de población rural (\%PR) y con necesidades básicas insatisfecha (\%NBI), y variables que describen la escala de las explotaciones ganaderas considerando la proporción de cabezas que pertenecen a rodeos $<200$ animales ( $R$ chicos) o rodeos $>1000$ animales ( $\mathrm{R}$ grandes) en el caso de bovinos, y de hatos < de 100 animales ( $\mathrm{H}$ chicos) o > de 200 animales ( $\mathrm{H}$ grandes) en el caso de caprinos, y finalmente variables que describen el uso de la tierra como fracción de área de pasturas, agricultura, y la tasa anual de desmonte. Se destacan en negrita las asociaciones significativas $(\mathrm{p}<0.01)$.

Table 1. Correlation coefficients and significance value for association between appropriation indicators of primary productivity and climatic, socioeconomic and productive variable. See details in legend in Spanish.

\begin{tabular}{lcccccc}
\hline & \multicolumn{8}{c}{ Vía de apropiación } \\
\hline \multirow{2}{*}{ Variables } & \multicolumn{1}{c}{ Ganadera } & \multicolumn{2}{c}{ Forestal } & \multicolumn{2}{c}{ Fuegos } \\
\cline { 2 - 8 } PPT & $\mathrm{r}^{2}$ & $\mathrm{p}$ & $\mathrm{r}^{2}$ & $\mathrm{p}$ & $\mathrm{r}^{2}$ & $\mathrm{p}$ \\
Lat & 0.02 & 0.7 & $\mathbf{0 . 2 9}$ & 0.01 & $\mathbf{0 . 5 8}$ & $<0.01$ \\
Dens & $\mathbf{0 . 3 6}$ & $<0.01$ & -0.21 & 0.08 & $\mathbf{- 0 . 3 0}$ & $<0.01$ \\
\%NBI & 0.01 & 0.94 & -0.07 & 0.58 & 0.01 & 0.93 \\
\%PR & -0.43 & $<0.01$ & 0.11 & 0.35 & 0.15 & 0.23 \\
R chicos & -0.03 & 0.80 & -0.01 & 0.4 & -0.12 & 0.33 \\
R grandes & $\mathbf{- 0 . 4 7}$ & $<0.01$ & 0.01 & 0.93 & -0.23 & 0.06 \\
H chicos & 0.28 & 0.02 & 0.06 & 0.63 & $\mathbf{0 . 3 7}$ & $<0.01$ \\
H grandes & $\mathbf{0 . 3 2}$ & $<0.01$ & 0.19 & 0.12 & $\mathbf{0 . 3 0}$ & $<0.01$ \\
Pasturas & -0.28 & 0.02 & -0.16 & 0.19 & -0.12 & 0.31 \\
Agricultura & $\mathbf{0 . 8 6}$ & $<0.01$ & -0.09 & 0.48 & 0.02 & 0.89 \\
Tasa desmonte & $\mathbf{0 . 6 6}$ & $<0.01$ & 0.03 & 0.8 & $\mathbf{0 . 3 0}$ & $<0.01$ \\
\hline & 0.31 & 0.12 & 0.26 & 0.18 & $\mathbf{0 . 6 5}$ & $<0.01$ \\
\hline
\end{tabular}

Considerando la totalidad del territorio, el fuego consumió en promedio $746 \mathrm{kgMS}$.ha${ }^{1}$.año ${ }^{-1}$ durante el período de análisis (20032006). La apropiación indirecta de la PPN aérea por fuego registró los máximos valores en el este de Santiago del Estero y en el sudeste de Salta (Figura 2d), con valores de 2660 a $3800 \mathrm{kgMS} \cdot \mathrm{ha}^{-1}$.año ${ }^{-1}$. Los valores mínimos se observan al sur de la región (La Rioja y algunos departamentos de Córdoba) y en el NE de Salta, con valores que van de 19 a $60 \mathrm{kgMS} \mathrm{ha}^{-1} \cdot \mathrm{anno}^{-1}$. La biomasa consumida por fuegos creció con la tasa de desmonte, hacia sectores con mayores precipitaciones $y$ en departamentos con predominancia de rodeos vacunos de $>1000$ cabezas (R grandes) y latitudes más bajas (Tabla 1).

El análisis de la relación entre las tasas anuales de desmonte y de consumo de biomasa por fuego, realizado exclusivamente para los departamentos de Santiago del Estero (la información de tasas de desmonte no estaba disponible para las demás provincias) permitió estimar la contribución relativa de los fuegos recurrentes que ocurren bajo coberturas estables vs. aquellas que acompañan por única vez al proceso de desmonte (Figura 4). La relación exponencial hallada indica que cuando la tasa de desmonte es cercana a cero el consumo de los fuegos se aproxima a 444 kgMS.ha ${ }^{-1} \cdot$ año $^{-1}$ (ordenada al origen, Figura 4), el promedio regional observado $(746$ kgMS.ha ${ }^{-1} \cdot a_{n} o^{-1}$ ) se elevaría entonces un $68 \%$ por encima de este valor como resultado del desmonte. Asumiendo que en promedio en las áreas que no están sujetas a desmonte la biomasa consumida por fuegos es la indicada más arriba, y considerando que todo lo que exceda este valor en los departamentos estudiados corresponde exclusivamente al área desmontada, puede estimarse la cantidad de biomasa que se quema en estas últimas áreas para cada departamento. Este ejercicio sugiere que la biomasa consumida por fuegos en áreas desmontadas de todo el Chaco Seco promedia los $\sim 42000 \mathrm{kgMS} \mathrm{ha}^{-1}$.

Según el modelo bioclimático de Del Grosso et al. (2008), la vegetación natural en el Chaco Seco tiene una PPN aérea media de $~ 5750$ kgMS.ha ${ }^{-1} \cdot a_{n} o^{-1}$. Si se considera la apropiación directa forestal $\left(66 \mathrm{kgMS} \cdot \mathrm{ha}^{-1}\right.$. año $\left.^{-1}\right)$ y ganadera estimada para esta cobertura (184 kgMS.ha${ }^{1} . a_{n} \mathrm{O}^{-1}$, ordenada al origen de la figura 3 ), la misma alcanza solamente un $4.3 \%$ y en conjunto con la indirecta por fuego elevarían el consumo anual de biomasa al $12.1 \%$. La figura 1 presenta una apropiación algo más alta (13\%) dado que

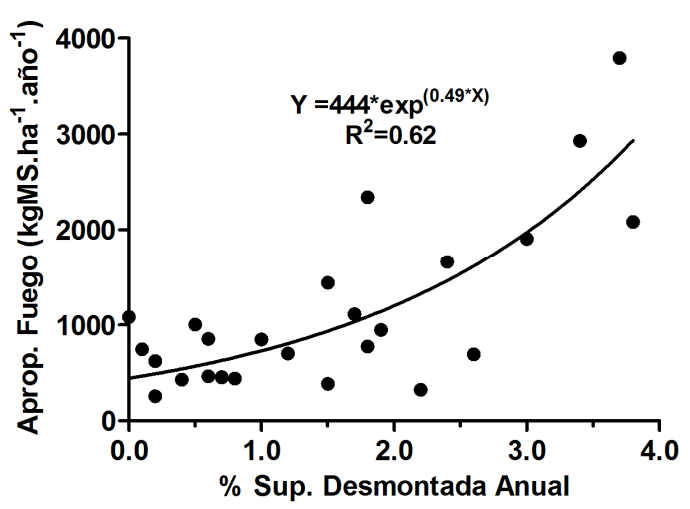

Figura 4. Relación entre la tasa anual de consumo de biomasa por fuego y la tasa anual de desmonte exclusivamente para los departamentos de la provincia de Santiago del Estero (período 2002-2006).

Figure 4. Relationship between the annual rate of biomass consumption by fires and the annual rate of forest clearing for exclusively the counties of Santiago del Estero province (period 2002-2006). 


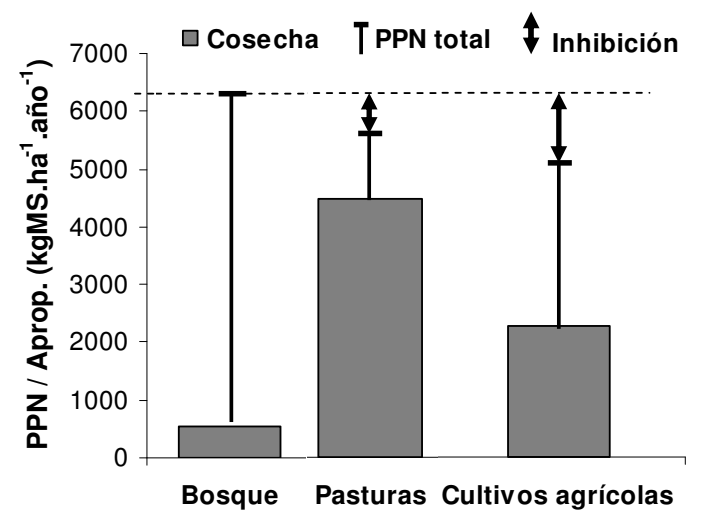

Figura 5. Estimación de la PPN aérea y su apropiación por cosecha bajo cobertura de bosque, pastura y cultivo agrícola en departamentos del Chaco Seco en los que estos tipos de vegetación y uso coexisten (Figura A2b). Las estimaciones de PPN aérea se basan en el modelo de Del Grosso et al. (2008) para bosques, en mediciones de campo (Cornacchione 2009, Kunst et al. 2006) para pasturas, y en datos de rendimiento reportados en censos anuales (SIIA 2012) afectados por un índice de cosecha de 0.45 (Satorre et al. 2004) para cultivos. La apropiación por cosecha ganadera surge del análisis de la Figura 3 (ordenada al origen para bosques, proyección al $100 \%$ de pastura para pasturas). La apropiación absoluta agrícola corresponde al cociente de la producción total reportada vs. el área total sembrada. Las flechas dobles indican la PPN que sería apropiada por inhibición en pasturas y cultivos agrícolas.

Figure 5. Aboveground NPP estimates and their direct appropriation through harvesting under forests, pastures, and annual crops in the Dry Chaco counties where such land covers coexist. See details in legend in Spanish.

considera el conjunto de la vegetación natural y el área cubierta por pasturas.

El análisis comparativo de la PPN aérea y su apropiación por cosecha bajo vegetación natural, pasturas y agricultura, focalizado en los 29 departamentos que comparten estas coberturas mostró una máxima apropiación para pasturas $(85 \%)$, intermedia para agricultura (45\%) y mínima para la vegetación natural $(9 \%)$. Se utilizó la relación hallada entre apropiación ganadera y porcentaje de pasturas (Figura 3) para definir los valores de apropiación ganadera de estas últimas y de la vegetación natural. La apropiación inhibitoria de la PPN aérea bajo vegetación implantada no superaría el 20\% (Figura 5).

\section{DisCUSIÓN}

El Chaco Seco muestra bajos valores de apropiación por cosecha de la producción primaria neta (PPN) en comparación con otros sistemas no agrícolas de ambientes semiáridos. Globalmente, la cosecha de productos ganaderos y forestales en estos sistemas alcanza valores medios de entre
820 y 960 kgMS.ha ${ }^{-1} \cdot$ año $^{-1}$, respectivamente (Haberl et al. 2007), mientras que en el área no agrícola del Chaco Seco se alcanzaría solo un 30 y $7 \%$ de cada uno de estos valores globales, respectivamente.

La apropiación de la PPN por cosecha varía intensamente con los cambios de cobertura de la tierra del Chaco Seco. En el caso de la cosecha ganadera, la misma se multiplica por un factor mayor a 20 cuando los bosques se transforman en pasturas (Figura 3). Este valor sugerido se acerca a la PPN aérea reportada para pastos en abras (pastizales naturales dentro del bosque) o pasturas implantadas (4000-5000 kgMS.ha- ${ }^{-1} \cdot$ año $^{-1}$, Kunst et al. 1999). El más fácil acceso al forraje y la mayor fracción de PPN destinada a tejidos con valor forrajero en las pasturas son importantes determinantes de estas diferencias pero no los únicos que deben considerarse (Kunst et al. 2003; Garbulsky \& Deregibus 2005). El establecimiento de pasturas suele ser acompañado también por mejoras de infraestructura, especialmente importantes en el caso de la oferta de agua de bebida al ganado (Nazar Anchorena 1988), que pueden ser igualmente relevantes explicando este dramático aumento de la capacidad de cosechar la PPN de esta región. La cosecha forestal actual de los sistemas de bosque no llega a compensar la baja cosecha ganadera que experimentan respecto a las pasturas, remarcando el bajo aprovechamiento productivo integral de la vegetación natural o seminatural en esta región (Figura 1). La fracción de la cosecha forestal máxima llega a extraer entre el 10 y $20 \%$ de las existencias de biomasa (Bonino 2006), solo el $20 \%\left(<10 \mathrm{kgMS} \cdot \mathrm{ha}^{-1}\right.$.año $\left.{ }^{-1}\right)$ es destinado al uso maderero, y éste valor es inferior al promedio global de $45 \%$ (MEA 2003).

En aquellos departamentos en los que es significativa el área cubierta por cultivos agrícolas, también se incrementa notablemente la apropiación de la PPN por cosecha bajo este uso (Figura A2). Si bien estas comparaciones sugieren que la transformación de la vegetación natural en el Chaco Seco provee grandes beneficios en la generación de bienes de valor comercial, es importante considerarlos en conjunto con otros servicios que el territorio brinda a la sociedad. Especialmente relevantes son la regulación hidrológica y el control del transporte de sales (Jobbágy et al. 2008), el almacenamiento de carbono (Grau et al. 2005, Gasparri et al. 2010), y la conservación de hábitats y biodiversidad (Zak et al. 2004; Galetto et al. 2007) que ofrece la vegetación natural. El análisis conjunto de estas funciones 
requiere criterios amplios en el diseño de sistemas productivos alternativos y en su evaluación comparativa (Laterra et al. 2012).

La importancia del fuego en el Chaco Seco como vía de consumo de la PPN es notable cuando se la compara con los valores de cosecha, siendo superior a los niveles globales de apropiación de la PPN por fuegos descriptos para los ecosistemas forestales del mundo (240 - 260 kgMS.ha- ${ }^{-1}$. año ${ }^{-1}$, Lauk \& Erb 2009). El análisis territorial de la disipación de biomasa por fuegos en la región sugiere que una parte considerable de las mismas es impulsada por el reemplazo de la vegetación natural por pasturas y cultivos. Los valores tan altos de disipación en desmontes destacan el bajísimo aprovechamiento del material forestal retirado por los desmontes. Si bien no fue posible desagregar la contribución a la disipación de PPN por fuegos que hacen las tierras que ya están bajo uso agrícola en el período de nuestro análisis, estudios previos sugieren que en regiones semiáridas, incluyendo al Chaco Seco, la agricultura reduciría la incidencia de fuegos una vez establecida (Di Bella et al. 2006, Fischer et al. 2012). La mayor apropiación por cosecha aportada por el establecimiento de pasturas o cultivos en el Chaco Seco es acompañada por una pequeña apropiación inhibitoria de la PPN que no superaría el 20\% (Figura 5), sin embargo debe considerarse en este análisis la apropiación disipativa vinculada al proceso de desmonte que involucra un episodio único pero intenso que podría consumir una cantidad de biomasa equivalente a la PPN aérea acumulada durante una década bajo vegetación natural.

ElChacoSeco mostró una alta heterogeneidad interna en relación a la magnitud de las distintas vías de apropiación de la PPN aérea (Figura 2). En el caso de la ganadería bovina la cosecha incrementó su magnitud absoluta en respuesta al área cultivada con pasturas (Figura 3) y agricultura, y disminuyó donde las poblaciones son más pobres y predominan las explotaciones ganaderas más pequeñas (Tabla 1, Información Suplementaria, Figura A3). Cabe destacar que, a pesar de tratarse de una región semiárida, se encontró solamente una relación marginal entre el consumo ganadero absoluto y la precipitación. Otro aspecto que caracteriza a la producción ganadera del Chaco Seco es el alto desvió a mantenimiento, producto de las bajas tasas reproductivas de su rodeo vacuno ( $45 \%$ de madres sin cría en el Chaco Seco vs. 11\% en la región pampenana hace más de dos décadas, Carrillo 1988).
A nivel global se ha planteado que las condiciones socioeconómicas exceden la influencia del clima determinando la productividad agrícola y ganadera de bosques secos subtropicales similares al Chaco (en Australia, África, Asia y Norteamérica) (Baldi y Jobbágy 2012, Baldi et al. 2012). Al focalizarnos en una única región y país, nuestro análisis destaca el papel de las diferencias en el desarrollo socioeconómico local modulando la apropiación de la PPN, remarcando que bajo condiciones de mayor pobreza la intensidad de uso e intervención sobre los ecosistemas se reduce (Baldi et al., 2012).

La precipitación fue, en cambio, la única variable que explicó las diferencias en los niveles de apropiación de la PPN aérea por cosecha de productos forestales, posiblemente como resultado de productividades de madera mayores y presencia de rodales de mayor valor para la producción de carbón vegetal hacia departamentos más húmedos. Es notable en este caso la baja correlación entre la cosecha forestal y el desmonte (evaluada sólo en Santiago del Estero), sugiriendo que éste no es acompañada por un uso efectivo de los residuos leñosos que genera. Finalmente, en el caso de la disipación de PPN aérea por fuego, cabe destacar que no solo las tasas de desmonte surgieron como determinante importante, sino también la presencia de rodeos vacunos grandes. Esto último se podría asociar a la práctica de quema prescripta y el posible efecto positivo de la precipitación y la temperatura (subyacente en el efecto negativo de la latitud) que combinarían mayor oferta de biomasa combustible y mejores condiciones para su combustión (Tabla 1).

El análisis presentado plantea oportunidades y desafíos para la conservación de los bosques del Chaco Seco en un contexto de creciente presión o demanda de bienes de los ecosistemas. La baja eficiencia con la que se aprovecha la vegetación natural de la región, tanto ganadera como forestalmente, puede favorecer su reemplazo por sistemas con mayor generación de bienes comercializables, como pasturas y agricultura (Figura 5). Los beneficios económicos que reportan estos sistemas alternativos son suficientemente altos para cubrir en poco tiempo sus costos de establecimiento y justificar los mayores riesgos y fluctuaciones que presentan, especialmente en el caso de los cultivos de secano. El alto consumo de biomasa por fuego sería un emergente de esta baja eficiencia productiva e incentivo para el reemplazo de la vegetación 
natural. Dentro del Chaco Seco se encuentra un gradiente que va desde situaciones de baja eficiencia productiva, pequeña escala de producción ganadera y altos índices de pobreza a otras de producción ganadera de mayor escala y alta conversión a pasturas. Este gradiente parece ser el resultado del contexto socioeconómico y en mucha menor medida del climático. Encontrar nuevas direcciones de cambio productivo en la región capaces de reconciliar estructuras de producción pequeñas, mantenimiento de la cubierta boscosa y apropiaciones mayores y sustentables de la PPN es uno de los desafíos más importantes para la población rural y los bosques de esta vasta región.

Agradecimientos: Este trabajo fue apoyado por el Programa de Becas AVG-CONICET, subvenciones del Centro Internacional de Investigación para el Desarrollo (IDRC - Canadá, el Proyecto 106601 - 001), el Instituto Interamericano para la Investigación del Cambio Global (IAI, CRN II 2031, National Science Foundation) y la ANPCyT (PRH 27 PICT 2008-00187). Damos las gracias a M Rueda, E Florio, FlLohaiza, A Gómez, M Navall, S Ballesteros, M Puente, R Gimenez, J Houspanossian, F Murray y $C$ López que proporcionaron una valiosa ayuda durante todo el estudio. Agradecemos a la editora, A Cingolani, y a dos revisores anónimos por sus valiosos aportes y generoso esfuerzo.

\section{BIBLIOGRAFIA}

AKaIKe, H. 1974. A new look at the statistical model identification. IEEE Transactions on Automatic Control, 19:716-723.

AnRIQueZ, A; A Albanesi; C Kunst; R Ledesma; C LóPez et al. 2005. Rolado de fachinales y calidad de suelos en el Chaco Occidental, Argentina. Ciencia del Suelo, 23:145-157.

Baldi G, S Verón \& EG Jobbágy. 2012. The imprint of humans on landscape patterns and vegetation functioning in the dry subtropics. Global Ecol. Biogeogr., doi: $10.1111 /$ gcb.12060

BALDI, G\& EG. JоввÁGy. 2012. Land use in the dry subtropics: Vegetation composition and production across contrasting human contexts. J. Arid Environ., 76:115-127.

Blanco, L; MO Aguilera; JM Paruelo \& FN Biurrun. 2008. Grazing effect on NDVI across an aridity gradient in Argentina. J. Arid Environ, 72:764-776.

BOND, W \& J KeELER. 2005. Fire as a global 'herbivore': the ecology and evolution of flammable ecosystems. Trends Ecol. Evol., 20:387-394.

BONINO, E. 2006. Changes in carbon pools associated with a land - use gradient in the Dry Chaco, Argentina. For. Ecol. Manag., 223:183-189.

Bravo, S; C KunSt; R Grau; \& E ArÁoz. 2010. Fire-rainfall relationships in Argentine Chaco savannas. J. Arid. Environ., 74:1319-1323.

BRown, A; \& S PACHelo. 2006. Propuesta de actualización del mapa ecorregional de la Argentina. En: Brown, A; U Martínez Ortiz; M Acerbi \& J Corchera. 2006. Editores. La Situación Ambiental Argentina 2005, FVSA Buenos Aires.

Bucher, E.1982. Chaco and Caatinga: South American arid savannas, woodlands and thickets. En: Huntley B \& B Walker (eds). Springer-Verlag, Germany. Ecol. Trop.
Savannas, 48-79 pp.

BUCINI, G \& NHANAN. 2007. A continental-scale analysis of tree cover in African savannas. Global Ecol. Biogeogr., 16:593-605.

CARRANZA, C \& M LedESMA. 2005. Sistemas silvopastoriles en el Chaco Árido. IDIA XXI Forestales. INTA 8:240-246.

Carranza, CA; L Pietrarelli, M Ledesma \& M Balzarini. 2000. Crecimiento de Prosopis flexuosa DC. En el Chaco Árido Argentino, luego de la Eliminación del Estrato Arbustivo. Multiequina, 9:119-133.

Carrillo, J. 1988. Manejo de un rodeo de cría. INTA. Ed. Hemisferio Sur Buenos Aires, 194 pp.

Chidumayo, EN \& DJ Gumbo. 2010. The dry forests and woodlands of Africa: Managing for products and services. Earthscan Forest Library 288 pp.

Cingolani, A; I Noir-Meir; D Renison \& M Cabido. 2008. La ganadería extensiva, ¿es compatible con la conservación dela biodiversidad y de los suelos? Ecología Austral, 18: 253-271.

CORnACCHIONE, M. 2009. Producción primaria de pasturas cultivadas. Aplicación de leyes provinciales 6.841 y 6.942. Jornadas de actualización y capacitación. CPIA, Santiago del Estero, Argentina.

Del Grosso, S; W Parton; TStohlgren; DZheng;DBachelet ET AL. 2008. Global potential net primary production predicted from vegetation class, precipitation, and temperature. Ecology, 89:2117-2126.

Di Rienzo, JA; F CASANOves, MG BalzarinI, L GonZALEZ, M TABLADA \& C Robledo. 2009. Grupo InfoStat, FCA, Universidad Nacional de Córdoba, Argentina

DiBella, CM; EG Jobbágy; JM Paruelo \& S PinNock. 2006. Continental fire density patterns in South America. Global Ecol. Biogeogr., 15:192-199.

Dirección de Bosques de la Nación. 2006. Anuario de Estadística Forestal Especies Nativas 1999 - 2006. Departamento de Estadística Forestal, Dirección de Bosques, Secretaría de Ambiente y Desarrollo Sustentable. ISSN 1850-7239. Versión digital.

Erb, K; F Krausmann; V Gaube; S Gingrich; A Bondeau ET AL. 2009. Analyzing the global human appropriation of net primary production: processes, trajectories, implications. An introduction. Ecol. Econ., 69:250-259.

FAO. 1983. Métodos simples para fabricar carbón vegetal. Serie de estudio FAO: Montes 41. Roma. En: http:/ / www.fao.org/docrep/X5328S/X5328S00.htm.

Fischer MA, CM DiBella \& EG JobBágy. 2012. Fire patterns in central Argentina. J. Arid Environ., 71:115-127.

Galetto L, R Aguiar, M Musicante, J Astegiano, A Ferreras, M JAUSORO, ET AL. 2007. Fragmentación de hábitats, riqueza de polinizadores, polinización y reproducción de plantas nativas en el Bosque Chaqueño de Córdoba, Argentina. Ecología Austral, 17: 67-80.

Garbulsky, M \& A Deregibus. 2005. Perfiles por país del recurso pastura/forraje. Argentina: uso de la tierra, topografía, clima, regiones ecológicas, recursos forrajeros, oportunidades para el mejoramiento de recursos forrajeros. FAO Roma. http://www.fao.org/ ag/grassland.htm.

GaSPARri,I;HGrau\&EMANGHI. 2008. Carbon poolsandemissions from deforestationin extra-tropical forests of northern Argentina between 1900 and 2005. Ecosystems, 11:1247-1261.

Gasparri, I; M Parmuchi; J Bono; H Karszenbaum \& C Montenegro. 2010. Assessing multi-temporal Landsat 7 ETM+ images for estimating above-ground biomass in subtropical dry forests of Argentina. J. Arid Environ., 74: 1272-1270.

Grau, H; M Aide \& I Gasparri. 2005. Globalization and Soybean Expansion into Semiarid Ecosystems of 
Argentina. Ambio, 34: 265-266.

GRAU,H;IGASPARRI \& M Aide. 2008. Balancing food production and nature conservation in the Neotropical dry forests of northern Argentina. Glob. Change Biol., 14:985-997.

HABERL, H. 2001. The energetic metabolism of societies part I: Accounting concepts. J. Ind. Ecol., 5:11-33.

HABERL, H. 2002. The energetic metabolism of societies part ii: Empirical examples. J. Ind. Ecol., 5:71-88.

HABERL, H. 2006. The global socioeconomic energetic metabolism as a sustainability problem. Energy, 31:87-99.

HaberL, H; K ERB; F KrausmanN; V Gaube; A Bondeau et al. 2007. Quantifying and mapping the human appropriation of net primary production in earth's terrestrial ecosystems. Proc. Natl. Acad. Sci. USA, 104:12942-12947.

Haberl, H; M Wackernagel \& T WrbKa. 2004. Land use and sustainability indicators. An introduction. Land Use Policy, 21:193-198.

IMHOFF, M; BOUNOUA, L; RICKETTS, T; LOUCKS, C; HARRISS, R \& LAWRENCE, W. 2004. Global patterns in human consumption of net primary production. Nature, 429:870-873.

INDEC. Instituto Nacional de Estadísticas y Censos. 2001. Censo Nacional de Población, Hogares y Viviendas 2001. Disponible en: http://www.indec.mecon.ar/ webcenso/index.asp

INDEC. Instituto Nacional de Estadísticas y Censos. 2002. Censo Nacional Agropecuario. Disponible en: http:/ / www.indec.mecon.ar/webcenso/index.asp

Jobbágy, E; M Nosetto; C SAntoni \& G Baldi. 2008. El desafío ecohidrológico de las transiciones entre sistemas leñosos y herbáceos en la llanura Chaco-Pampeana. Ecología Austral, 18:305-322.

JORGENSEN, SE. 2002. Integration of ecosystem theories: a pattern. Springer. $420 \mathrm{pp}$.

Krausmann, F; K Erb; S Gingrich; C Lauck \& H Haberl. 2007. Global patterns of socioeconomic biomass flows in the year 2000: a comprehensive assessment of supply, consumption and constraints. Ecol. Econ., doi: 10.1016/ j.ecolecon.2007.07.012

Kunst, C; R Renolfi \& A Fumagalli. 1999. Habilitación de tierras en el Chaco Semiárido Santiagueño. Memorias 2da Jornada Ganadera del NOA. Proyecto Ganadero Macrorregional INTA. Salta. 125 p.

Kunst, C; S Bravo; F Moscovich; J Herrera; J Godoy et AL. 2003. Fecha de aplicación de fuego y diversidad de herbáceas en una sabana de Elionorus muticus (Spreng) O. Rev. Chil. Hist. Nat., 76:105-115.

Kunst, C; E Monti; H Peres \& J Godoy. 2006. Assessment of the rangelands of southwestern Santiago del Estero, Argentina, for grazing management and research. J. Environ. Manag., 80:248-265.

LATERra P; E JobBagy \& J Paruelo. 2011. Valoración de servicios ecosistémicos. Conceptos, herramientas y aplicaciones para el ordenamiento territorial. INTA p:741.

LAUK, C \& K ERB. 2009. Biomass consumed in anthropogenic vegetation fires: Global patterns and processes. Ecol. Econ., 69: 301-309

Mariot, V; A Reuter; A Palavecino \& F Zubrinic. 2007. Determinación multitemporal del uso del suelo en la provincia de santiago del estero hasta el año 2007. Proyecto de Investigación FCF-UNSE.

Martínez Alier, J. 1998. Curso de economía ecológica. Programa de las Naciones Unidas para el Medio Ambiente. Oficina Regional para América Latina y el Caribe. Serie Textos Básicos para la Formación Ambiental 1.

MillenNium Ecosystem Assessment (MEA). Ecosystems And
HUMAN WELL-BEING: CURRENT STATE AND TRENDS: FINDINGS OF THE CONDITION AND TRENDS WorKING. 2003. Group edited: Hassan R, Scholes R and Ash N. The millennium ecosystem assessment series 1. USA. ISBN 1-55963-227-5.

Miñon D, A. Fumagalli, \& A. Auslender. 1991. Hábitos alimentarios de vacunos y caprinos en bosque de la región chaqueña semiárida. Rev. Arg. Prod. Animal, 11:275-283.

Navarrete, M; G Gallopin; M Blanco; M Diaz-Zorita; D FERRARO ET AL. 2005. Análisis sistémico de la agriculturización en la pampa húmeda argentina y sus consecuencias en regiones extrapampeanas: sostenibilidad, brechas de conocimiento $e$ integración de políticas. Serie Medio Ambiente y Desarrollo-CEPAL, 65:118 pp.

NAZAR ANCHORENA, JB. 1988. Pastizales Naturales de La Pampa. Tomo II Convenio AACREA - Prov. de La Pampa. 112 pp.

ODUM, HT. 2002. Explanations of ecological relationships with energy systems concepts. Ecol. Model., 158:201-211.

Oesterheld, M; E SAla \& S McNaughton. 1992. The effect of animal husbandry on herbivore-carrying capacity at a regional scale. Nature, 356:234-236.

Oesterheld, M; CM Di Bella \& H Kerdiles. 1998. Relation between NOAA-AVHRR satellite data and stocking of rate rangelands. Ecol. App., 8:207-212.

Paruelo, JM; JP Guerschman; G Piñeiro; EG Jobbágy et AL. 2006. Cambios en el uso de la tierra en Argentina y Uruguay: marcos conceptuales para su análisis. Agrociencia, 2:47-61.

Rojstaczer, S; S Sterling \& N Moore. 2001. Human appropriation of photosynthesis products. Science, 249: 2549-2552.

Quiroga, E; L Blanco \& E Orionte. 2009. Evaluación de estrategias de rehabilitación de pastizales áridos. Ecología Austral, 19:107-117.

Sala, O; W Parton; L Joyce \& W Lauenroth. 1988. Primary production of the central grassland region of the United States. Ecology, 69:40-45.

Satorre E, R Benech Arnold; G Slafer; E de la Fuente;D Miralles et AL. 2004. Producción de Granos. Bases funcionales para su manejo. Editorial Facultad Agronomía UBA. 783 pp

SCHLESINGER, W. 1997. Biogeoquímica. Un análisis del cambio global. Ariel Ciencia 2000. España. 592 pp.

SEABrook, L; C McAlpine \& R Fensham. 2006. Cattle, crops and clearing: Regional drivers of landscape change in the Brigalow Belt, Queensland, Australia, 1840-2004. Landscape Urban Plan., 78:373-385.

SIIA. SistemA DE INTEGRADO DE INFORMACIÓN AGROPECUARIA. 2012. Ministerio de Agricultura, Ganadería y Pesca. Presidencia de la Nación. Disponible en: http://www.siia.gov.ar/ index.php/series-por-tema/agricultura.

TINKLER, DE. 2004. Historical change in crop distribution in Texas and its potential application for modeling wildlife distributions. Texas Tech University, 90 pp.

VAN DAM, C. 1999. Cambio tecnológico, concentración de la propiedad y desarrollo sostenible. Los efectos de la introducción del paquete soja/siembra directa en el Umbral al Chaco. Debate Agrario, 35:133-181.

Verón, SR, EG JobBÁgy, CM Di Bella, JM PARUelo \& RB JACKSON. 2012. Assessing the potential of wildfires as a sustainable bioenergy opportunity. Glob. Change Biol. Bioenergy, doi: 10.1111/j.1757-1707.2012.01181.x.

VitouseK, P; P Ehrlich; A Ehrlich; \& P MAtson. 1986. Human appropriation of the products of photosynthesis. BioScience, 36:368-373.

ZAK, M; M CABIDO \& J Hodgson. 2004. Do subtropical seasonal forests in the Gran Chaco, Argentina, have a future? Biol. Cons., 120:589-598. 


\section{INFORMACIÓN SUPLEMENTARIA}

\section{ANEXO 1: INDICADORES DE APROPIACIÓN HUMANA}

\section{Apropiación Ganadera}

Se utilizaron los datos censales de existencias de ganado por departamento. En el caso de vacunos las categorías reportadas se discriminaron en vacas con cría y sin cría (correspondiendo la primera al número de terneros y al segunda al número de vacas menos el de terneros), terneros, vaquillonas, novillos, novillitos, toritos, toros y sin definir. A cada una de estas categorías se le asignó un peso vivo de 400, 100, 200, 350, 250, 350, 600 y $100 \mathrm{~kg}$, respectivamente. En el caso de las categorías vaquillona, novillo, novillito, torito se consideró una ganancia diaria de peso de $0.1 \mathrm{Kg}_{\text {.dia-1 }}$. En el caso de los cálculos de consumo de terneros, los requerimientos se computaron en forma diferencial para el período de gestación (120 días), lactancia (90 días) y crecimiento (90 días.

Para el ganado caprino se discriminaron las categorías cabra, cabrilla, cabrito, capón, reproductor y no categorizado, con pesos vivos supuestos de 25, 15, 6, 30, 40 y 6 kg; respectivamente. Se utilizaron las ecuaciones del NRC (1981) citadas por Elizondo (2002) para determinar requerimientos energéticos para mantenimiento, gestación, lactancia y crecimiento. En la categoría madre se computaron valores de energía extra por gestación para los dos últimos meses y lactancia por 45 días por ciclo. Asumiendo un potencial de 1.5 pariciones por madre por año, se cálculo el número de cabras con y sin cría en función del número de cabritos/1.5 y el número de cabras menos el número de cabritos/1.5, respectivamente. Se consideró para la región un contenido de grasa en leche caprina de 5.5\% (Javier Ponce, com. pers.).

A partir de las características mencionadas se determinó el valor de energía neta de mantenimiento (EN) requerido por cada especie y categoría y a partir de ese valor se (i) discriminó aquella destinada a la producción (ENP) y al mantenimiento animal (ENM) según la proporción de animales juveniles y madres con cría vs. madres sin cría y machos adultos, respectivamente; y se calculó (ii) la energía metabólica $(\mathrm{EM}=\mathrm{EN} / \mathrm{k}$, donde $\mathrm{k}$ es el incremento calórico según $\mathrm{k}=0.35 \mathrm{q}$ +0.5 , siendo $q=0.82^{*} \mathrm{D} / 100$ en donde $\mathrm{D}$ es la digestibilidad del forraje), (iii) la energía digestible $(\mathrm{ED}=\mathrm{EM} / 0.82)$, y (iv) la energía bruta $(\mathrm{EB}=\mathrm{ED} /$ digestibilidad / 100 ) según lo propuesto por Cangiano (1996), asumiendo una digestibilidad media del forraje consumido de 55\% (Kunst et al. 1999, 2003; Garbulsky et al. 2005). Estos cálculos se apoyaron en las ecuaciones de las tablas A1.1 y A1.2 y los valores fueron convertidos a unidades de materia seca por unidad de superficie $\left(\mathrm{kgMS} \cdot \mathrm{ha}^{-1} \cdot \mathrm{anno}^{-1}\right)$, considerando una equivalencia de $4.18 \mathrm{kgMS} / \mathrm{Mcal}$.

Tabla A1.1. Ecuaciones utilizadas para el cálculo de los requerimientos energéticos del ganado bovino, en unidades de Mcal.día ${ }^{-1}$. Siglas: Concentración Energética (CE), Requerimientos de energía por: mantenimiento (REM), lactancia (RL), por gestación (RG), de Terneros (RT), y de ganancia de peso (RGP).

Table A1.1. Equations used for the calculation of the energetic requirements of cattle, in units of Mcal.day ${ }^{-1}$. Abbreviations: Energy Concentration (CE), Requirements of energy for: Maintenance (REM), of lactation (RL), gestation (RG), calf (RT) and weight gain (RGP).

\begin{tabular}{|c|c|c|c|}
\hline Variables & Ecuaciones & Observaciones & Fuente \\
\hline $\begin{array}{l}\text { Concentración energética } \\
\text { (CE) }\end{array}$ & $C E=0.18+0.038 D$ & $\begin{array}{l}\text { D es porcentaje de } \\
\text { digestibilidad del forraje, } \\
\text { asumida en } 55 \%\end{array}$ & $\begin{array}{l}\text { Rittenhouse et al. } \\
\text { (1971) }\end{array}$ \\
\hline $\begin{array}{l}\text { Requerimientos de energía } \\
\text { de mantenimiento (REM) }\end{array}$ & $\mathrm{REM}=7.2-1.6 \mathrm{CE}+0.02 \mathrm{PV}$ & Peso Vivo (PV) & \multirow{5}{*}{$\begin{array}{l}\text { Adaptadas } \\
\text { de tablas y } \\
\text { ecuaciones de } \\
\text { Geenty \& Rattray } \\
\text { (1987) citadas } \\
\text { por Cangiano } \\
\text { (1996). }\end{array}$} \\
\hline $\begin{array}{l}\text { Requerimientos extras por } \\
\text { lactancia }(R L)^{*}\end{array}$ & $\begin{array}{l}\mathrm{RL}=0.048+0.81 \mathrm{ML}+ \\
0.042 \mathrm{PV}\end{array}$ & $\begin{array}{l}\text { Mes de lactancia (ML) varía } \\
\text { de } 1 \text { a } 3 .\end{array}$ & \\
\hline $\begin{array}{l}\text { Requerimientos extras por } \\
\text { gestación }(\mathrm{RG})^{*}\end{array}$ & $R G=10.17 \mathrm{e}^{(-0.5439 \mathrm{M})}$ & Mes (M) varía entre 3 y 0. & \\
\hline $\begin{array}{l}\text { Requerimientos energéticos } \\
\text { diario de terneros (RT) }\end{array}$ & $\begin{array}{l}\mathrm{RT}=1.2 \mathrm{MV}^{2}-1.2 \mathrm{MV} \\
-1.10^{-13}\end{array}$ & \multirow{2}{*}{$\begin{array}{l}\text { Mes de vida }(\mathrm{MV}) \text {, se } \\
\text { incluyen el } 2^{\circ} \text { y el } 3^{\circ} \text {. } \\
\text { Ganancia diaria }(\mathrm{GD})=0.1 \\
\mathrm{~kg} \cdot \text { día }^{-1} \text {. }\end{array}$} & \\
\hline $\begin{array}{l}\text { Requerimientos diario de } \\
\text { ganancia de peso (RGP) }\end{array}$ & $\mathrm{RGP}=0+7.04 \mathrm{GD}+0.04 \mathrm{PV}$ & & \\
\hline
\end{tabular}


Tabla A1.2. Ecuaciones usadas para el cálculo de los requerimientos energéticos del ganado caprino. Fuente: NRC (1981), citadas por Elizondo (2002), todas en unidades se expresan en kgMS.ha-1. año ${ }^{-1}$. Siglas: Energía Digestible (ED), Energía Metabólica (EM), Energía Neta de Mantenimiento (ENM), Requerimientos extras de lactación (RL).

Table A1.2: Equations used for calculated the requirements energetic of goats. Source: NRC (1981), cited by Elizondo (2002), units expressed in kgMS.ha ${ }^{-1}$.year ${ }^{-1}$. Abbreviations: Digestible Energy (ED), Metabolic Energy (EM), Net Energy Maintenance (ENM), extra requirements of lactation (RL).

\begin{tabular}{lll}
\hline Variable & Ecuación & Observaciones \\
\hline Energía Digestible (ED) & $\mathrm{ED}=0.4927+0.0353 \mathrm{PV}$ & Peso Vivo (PV) \\
Energía Metabólica (EM) & $\mathrm{EM}=0.4000+0.0289 \mathrm{PV}$ & \\
Energía Neta de Mantenimiento (ENM) & $\mathrm{ENM}=0.2240+0.0163 \mathrm{PV}$ & \\
Requerimientos extras de lactación (RL) & $\mathrm{RL}=1.37+0.04 \mathrm{G}$ & $\mathrm{G}=$ porcentaje de grasa en leche. \\
\hline
\end{tabular}

\section{Apropiación Forestal}

Lo datos tomados para el cálculo de apropiación forestal son los registrados en unidades de masa de productos forestales cosechados por departamento, incluyendo leña, carbón, postes rodrigones, varillas, rollizos, y otros productos aserrados. Debido a que las estadísticas de la Dirección Nacional de Bosque no incluyen el consumo doméstico de energía en forma de leña o carbón vegetal, se utilizó información censal sobre número de hogares que usan leña como combustible para cocinar (INDEC 2001) y se consideró un consumo anual de $14000 \mathrm{~kg}$ de leña.hogar ${ }^{-1}$.año ${ }^{1}$ (Calvo et al. 2007). Para la biomasa leñosa total se consideró un extra por residuos de $10 \%$ para leña y carbón y 50\% para los productos restantes (Silvia Vélez, com. pers.).

\section{Apropiación por Fuego}

Verón et al (2012) utilizaron productos satelitales MOD y MYD 14CMG colección 4 (Giglio et al. 2006), que integran cuatro observaciones diarias de un $1 \mathrm{~km}$ de resolución espacial de los sensores Aqua y Terra a bordo de la plataforma MODIS en valores mensuales para celdas de $0.5 \times 0.5^{\circ}$. La energía radiativa de cada celda se calculó mediante una aproximación probabilística y fue validada con "Global Fire Emissions Database v3" (van der Werf 2010). Dado que la energía radiativa representa aproximadamente el $12.3 \%$ de la energía total (Wooster et al. 2005; Freeborn et al. 2007), la energía radiativa de los fuegos se multiplico por 8.1 para obtener la energía total disipada por el fuego. Por último, Verón et al (2012) obtuvieron la cantidad de biomasa quemada a partir del cociente entre la energía total disipada por el fuego y el valor calorífico de la biomasa vegetal (18 MJ.kg-1, Campbell et al. 2009). Superponiendo el mapa departamental a la base de datos de Verón et al (2012) se estimó a la escala regional, el promedio anual de energía disipada por fuegos para cada departamento.

\section{Bibliografía}

Calvo, S; L Salvador; R CoIrini; A Müller; N Reinoso \& A Visintini. 2007. Indicadores de sustentabilidad. Relaciones con la valoración económica del bosque nativo, Córdoba, Argentina. Zonas Áridas 11:32-46.

Campbell, JE; DB Lobell \& CB Field. 2009. Greater transportation energy and GHG offsets from bioelectricity than ethanol. Science 324:1055-1057.

ELIZONDO, J. 2002. Estimación lineal de los requerimientos nutricionales del NRC para cabras. Universidad de Costa Rica. Agron. Mesoamericana 3:159-163.

Geenty \& Rattray 1987, citado por Cangiano, CA. 1996. Producción Animal. INTA Balcarce, Argentina. 154 pp.

Giglio, L; GR Werf, J Randerson, G Collatz \& P KASIBHATLA. 2006. Global estimation of burned area using MODIS active fire observations. Atmos. Chem. Phys. 6:957-974.

Freeborn, PH; MJ Wooster; WM Hao; CA Ryan; B NordGREN ET AL. 2007. Relationships between energy release, fuel mass loss, and trace gas and aerosol emissions during laboratory biomass fires. J. Geophys. Res. 113: D01301.

WOOSTER, MJ; GRoBertS; GL PerRY \& J KAUfMAN. 2005. Retrieval of biomass combustion rates and totals from fire radiative power observations: FRP derivation and calibration relationships between biomass consumption and fire radiative energy release. J. Geophys. Res. 110: D24311.

Rittenhouse, LR; CL Streeter \& DC Clanton. 1971. Estimating digestible energy from digestible dry and organic matter in diets of grazing cattle. Technical Note. J. Range Manag. 24:73-75.

VAN DER WERF, GR; JT RANDERSON; L Giglio; G J COLlatZ; PS KASIBHATLA; \& AF ARELLANO. 2006. Interannual variability in global biomass burning emissions from 1997 to 2004 . Atmos. Chem. Phys. 6: 3423-3441. 


\section{ANeXo 2: DePARTAMENTOS DEL AREA DE ESTUDIO}
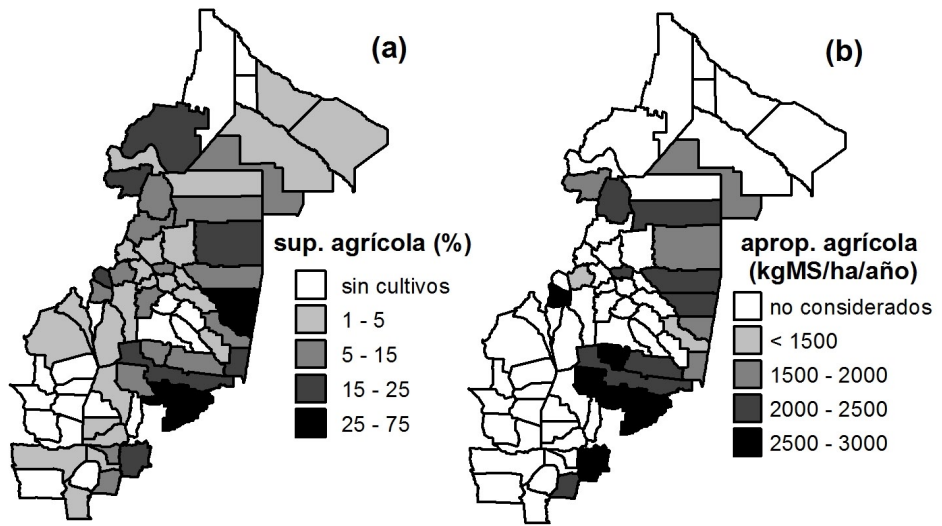

Figura A2. (a) Fracción de la superficie implantada con cultivos agrícolas. (b) Apropiación de la producción primaria neta aérea por cultivos de secano según rendimientos promedios $\left(\mathrm{kgMS} \mathrm{ha}^{-1}\right.$.año ${ }^{-1}$ ) para las campañas 2002-2006 (SIIA 2012). En este caso sólo se consideran los departamentos con $>2 \%$ de área agrícola y dentro de estos se descartan aquellos con una participación del riego $>3 \%$. Los valores de apropiación se refieren al área sembrada bajo secano.

Figure A2. (a) Fraction of the area devoted to agricultural crops. (b) Appropriation of aboveground net primary production under rainfed crops using average yields $\left(\mathrm{kgDM}_{\mathrm{h}} \mathrm{h}^{-1} . \mathrm{yr}^{-1}\right)$ for the 2002-2006 growing seasons (SIIA 2012). In these cases only those counties with more than $2 \%$ of the area with agricultural crops are considered and those with more than $3 \%$ of the agricultural area with irrigation are discarded. Appropriation values are referred to the non-irrigated sown area.

Tabla A2. Departamentos que comprenden el área de estudio, superficie, PPN áerea e indicadores de apropiación. Los valores de las diferentes apropiaciones se estimaron sobre las siguientes superficies a) apropiación ganadera relativa a la superficie natural + pastura, b) forestal sobre superficie natural, c) agricultura sobre superficie cultivada implantada y d) apropiación por fuego sobre superficie total. La suma de las tres clases de cobertura analizadas puede no incorporar el 100\% de la superficie departamental por la presencia de áreas urbanas o salinas.

Table A2. Counties comprised in the study area, aboveground NPP, appropriation indicators of primary productivity and total surface. the different values of appropriation to estimated over followed surface a) relative livestock appropriation on the grass and vegetation nature surface, b) logging on natural surface, d) agricultural on cultivated surface and d) fire over total surface. The sum of the three kinds of coverage may not incorporate $100 \%$ analyzed of the surface of counties due at presence of urban or saline areas.

\begin{tabular}{|c|c|c|c|c|c|c|c|c|c|c|c|}
\hline \multirow[b]{2}{*}{ Código } & \multirow[b]{2}{*}{ Departamento } & \multirow[b]{2}{*}{ Provincia } & \multirow[b]{2}{*}{$\underset{\left(\mathrm{km}^{2}\right)}{\text { Superfieie }}$} & \multirow[b]{2}{*}{$\begin{array}{l}\text { PPN áerea } \\
\left(\mathrm{Kg} \text { MS.ha- }^{-}\right. \\
\left.{ }^{1} \cdot \mathrm{anno}^{-1}\right)\end{array}$} & \multicolumn{3}{|c|}{ Cobertura (\%) } & \multicolumn{4}{|c|}{$\begin{array}{c}\text { Apropiación (Kg MS.ha- } \\
\left.{ }^{1} \cdot \mathrm{anno}^{-1}\right)\end{array}$} \\
\hline & & & & & 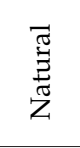 & 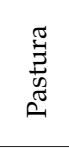 & 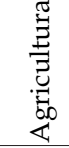 & $\begin{array}{l}\pi \\
\frac{\pi}{0} \\
\overparen{\pi} \\
\mathbb{0} \\
0\end{array}$ & 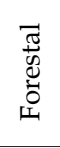 & $\begin{array}{l}\frac{\pi}{8} \\
\frac{0}{0} \\
\frac{0}{4}\end{array}$ & 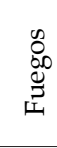 \\
\hline 1 & Anta & Salta & 22182 & 6247 & 81.3 & 2.8 & 15.9 & 230 & 24 & 2088 & 333 \\
\hline 2 & Metán & Salta & 54757 & 6098 & 98.4 & 0.2 & 1.4 & 24 & 3 & 1827 & 25 \\
\hline 3 & Rivadavia & Salta & 26383 & 5629 & 100.0 & 0.0 & - & 44 & 8 & 0 & 0 \\
\hline 4 & $\begin{array}{l}\text { Rosario de la } \\
\text { Frontera }\end{array}$ & Salta & 5349 & 5912 & 82.8 & 2.0 & 15.2 & 264 & 15 & 1811 & 275 \\
\hline 5 & Ayacucho & San Luis & 9425 & 3739 & 98.4 & 0.3 & 1.3 & 169 & 8 & 2890 & 37 \\
\hline 6 & Belgrano & San Luis & 7016 & 3852 & 99.8 & 0.2 & 0.0 & 186 & 2 & 867 & 0 \\
\hline 7 & Chacabuco & San Luis & 2830 & 5698 & 77.0 & 16.3 & 6.7 & 883 & 5 & 2366 & 159 \\
\hline 8 & Coronel Pringles & San Luis & 2195 & 5307 & 71.0 & 21.5 & 7.5 & 336 & 4 & 2917 & 219 \\
\hline 9 & Junín & San Luis & 4095 & 5018 & 98.6 & 1.0 & 0.4 & 673 & 1 & 2149 & 9 \\
\hline 10 & $\begin{array}{l}\text { Libertador General } \\
\text { San Martín }\end{array}$ & San Luis & 2969 & 5110 & 97.6 & 2.3 & 0.0 & 505 & 1 & 0 & 0 \\
\hline 11 & Ancasti & Catamarca & 2081 & 4465 & 99.9 & 0.1 & - & 178 & 2 & 0 & 0 \\
\hline 12 & Capayán & Catamarca & 4562 & 4147 & 98.9 & 0.9 & 0.2 & 128 & 16 & 4500 & 10 \\
\hline 13 & El Alto & Catamarca & 2080 & 4811 & 86.0 & 0.1 & 14.0 & 721 & 55 & 3157 & 441 \\
\hline 14 & $\mathrm{LaPaz}$ & Catamarca & 7892 & 4206 & 68.4 & 7.9 & 0.3 & 572 & 89 & 1877 & 6 \\
\hline 15 & Santa Rosa & Catamarca & 1424 & 5378 & 73.8 & 5.5 & 20.7 & 845 & 164 & 3119 & 645 \\
\hline 16 & Calamuchita & Córdoba & 4668 & 6137 & 79.1 & 2.1 & 18.9 & 676 & 2 & 2733 & 516 \\
\hline 17 & Cruz del Eje & Córdoba & 6539 & 4655 & 75.0 & 4.2 & 0.2 & 410 & 10 & 2008 & 4 \\
\hline
\end{tabular}




\begin{tabular}{|c|c|c|c|c|c|c|c|c|c|c|c|}
\hline \multirow[b]{2}{*}{ Código } & \multirow[b]{2}{*}{ Departamento } & \multirow[b]{2}{*}{ Provincia } & \multirow[b]{2}{*}{$\begin{array}{c}\text { Superficie } \\
\left(\mathrm{km}^{2}\right)\end{array}$} & \multirow[b]{2}{*}{ 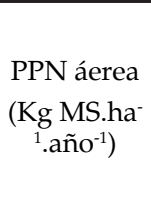 } & \multicolumn{3}{|c|}{ Cobertura (\%) } & \multicolumn{4}{|c|}{$\begin{array}{c}\text { Apropiación (Kg MS.ha- } \\
\left.{ }^{1} \cdot \mathrm{anno}^{-1}\right) \\
\end{array}$} \\
\hline & & & & & $\begin{array}{l}\bar{\pi} \\
\stackrel{\Xi}{\pi} \\
\text { Z }\end{array}$ & 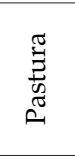 & 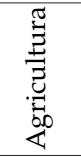 & 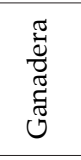 & 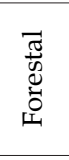 & 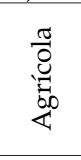 & 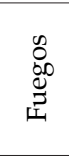 \\
\hline 18 & Ischilín & Córdoba & 5038 & 4937 & 62.4 & 10.2 & 13.0 & 750 & 26 & 2878 & 374 \\
\hline 19 & Minas & Córdoba & 3589 & 4507 & 99.5 & 0.5 & - & 208 & 2 & 0 & 0 \\
\hline 20 & Pocho & Córdoba & 3122 & 4760 & 95.3 & 1.1 & 3.6 & 288 & 3 & 2988 & 107 \\
\hline 21 & Punilla & Córdoba & 2616 & 5724 & 99.2 & 0.8 & - & 425 & 1 & 0 & 0 \\
\hline 22 & Río Primero & Córdoba & 6827 & 6612 & 14.1 & 12.7 & 73.2 & 2469 & 29 & 2683 & 1964 \\
\hline 23 & Río Seco & Córdoba & 6150 & 6873 & 76.2 & 9.6 & 14.3 & 634 & 16 & 2190 & 312 \\
\hline 24 & San Alberto & Córdoba & 3282 & 5257 & 97.1 & 1.7 & 1.2 & 358 & 11 & 3834 & 45 \\
\hline 25 & San Javier & Córdoba & 1583 & 5603 & 84.5 & 10.1 & 5.5 & 637 & 25 & 3620 & 198 \\
\hline 26 & Sobremonte & Córdoba & 3104 & 5637 & 80.4 & 4.7 & 8.5 & 595 & 5 & 3071 & 262 \\
\hline 27 & Totoral & Córdoba & 3190 & 6135 & 22.3 & 21.6 & 56.1 & 2638 & 21 & 2975 & 1670 \\
\hline 28 & Tulumba & Córdoba & 10369 & 6026 & 68.6 & 5.2 & 16.2 & 411 & 14 & 2286 & 370 \\
\hline 29 & Almirante Brown & Chaco & 17276 & 6481 & 92.4 & 0.7 & 6.9 & 151 & 564 & 1834 & 126 \\
\hline 30 & General Güemes & Chaco & 25487 & 6430 & 98.9 & 0.5 & 0.6 & 208 & 111 & 2253 & 14 \\
\hline 31 & Bermejo & Formosa & 12850 & 5426 & 99.9 & 0.0 & 0.1 & 214 & 15 & 2588 & 3 \\
\hline 32 & Matacos & Formosa & 4431 & 5242 & 100.0 & 0.0 & - & 118 & 16 & 0 & 0 \\
\hline 33 & Patiño & Formosa & 24502 & 7333 & 98.9 & 0.6 & 0.4 & 372 & 71 & 1650 & 7 \\
\hline 34 & Ramón Lista & Formosa & 3800 & 4843 & 100.0 & 0.0 & - & 124 & 7 & 0 & 0 \\
\hline 35 & Capital & La Rioja & 13638 & 3969 & 99.0 & 0.5 & 0.1 & 63 & 1 & 1212 & 1 \\
\hline 36 & Chamical & La Rioja & 5549 & 3893 & 80.2 & 0.9 & - & 142 & 1 & 0 & 0 \\
\hline 37 & $\begin{array}{l}\text { General Angel V. } \\
\text { Peñaloza }\end{array}$ & La Rioja & 3106 & 3816 & 99.9 & 0.1 & - & 143 & 11 & 0 & 0 \\
\hline 38 & General Belgrano & La Rioja & 2556 & 3943 & 99.5 & 0.5 & - & 263 & 4 & 0 & 0 \\
\hline 39 & $\begin{array}{l}\text { General Juan F. } \\
\text { Quiroga }\end{array}$ & La Rioja & 2585 & 3560 & 99.7 & 0.3 & - & 247 & 11 & 0 & 0 \\
\hline 40 & General Ocampo & La Rioja & 2135 & 3989 & 99.4 & 0.6 & - & 527 & 15 & 0 & 0 \\
\hline 41 & General San Martí & nLa Rioja & 5034 & 3960 & 99.5 & 0.5 & - & 200 & 43 & 0 & 0 \\
\hline 42 & $\begin{array}{l}\text { Rosario Vera } \\
\text { Peñaloza }\end{array}$ & La Rioja & 6114 & 3424 & 99.3 & 0.7 & - & 141 & 6 & 0 & 0 \\
\hline 43 & Aguirre & $\begin{array}{l}\text { Santiago del } \\
\text { Estero }\end{array}$ & 3378 & 6963 & 88.6 & 3.0 & 8.4 & 624 & 626 & 1856 & 155 \\
\hline 44 & Alberdi & $\begin{array}{l}\text { Santiago del } \\
\text { Estero }\end{array}$ & 12834 & 6281 & 93.3 & 0.2 & 6.4 & 70 & 91 & 2034 & 131 \\
\hline 45 & Atamisqui & $\begin{array}{l}\text { Santiago del } \\
\text { Estero }\end{array}$ & 2463 & 5592 & 99.9 & 0.1 & - & 134 & 16 & 0 & 0 \\
\hline 46 & Avellaneda & $\begin{array}{l}\text { Santiago del } \\
\text { Estero }\end{array}$ & 3640 & 6171 & 98.4 & 0.8 & 0.8 & 99 & 46 & 657 & 5 \\
\hline 47 & Banda & $\begin{array}{l}\text { Santiago del } \\
\text { Estero }\end{array}$ & 3991 & 5241 & 95.6 & 1.1 & 2.7 & 62 & 36 & 1934 & 53 \\
\hline 48 & Belgrano & $\begin{array}{l}\text { Santiago del } \\
\text { Estero }\end{array}$ & 3347 & 7095 & 46.1 & 6.7 & 47.2 & 929 & 5 & 2205 & 1041 \\
\hline 49 & Capital & $\begin{array}{l}\text { Santiago del } \\
\text { Estero }\end{array}$ & 2250 & 5082 & 45.0 & 1.4 & 0.2 & 297 & 61 & 1803 & 4 \\
\hline 50 & Choya & $\begin{array}{l}\text { Santiago del } \\
\text { Estero }\end{array}$ & 13094 & 6300 & 94.9 & 0.3 & 1.1 & 108 & 21 & 1704 & 19 \\
\hline 51 & Copo & $\begin{array}{l}\text { Santiago del } \\
\text { Estero }\end{array}$ & 6207 & 4805 & 95.9 & 0.2 & 3.9 & 187 & 240 & 1923 & 74 \\
\hline 52 & Figueroa & $\begin{array}{l}\text { Santiago del } \\
\text { Estero }\end{array}$ & 6413 & 5698 & 99.4 & 0.0 & 0.6 & 70 & 40 & 1272 & 8 \\
\hline 53 & General Taboada & $\begin{array}{l}\text { Santiago del } \\
\text { Estero }\end{array}$ & 6091 & 6993 & 58.8 & 3.5 & 37.8 & 436 & 146 & 2025 & 765 \\
\hline 54 & Guasayán & $\begin{array}{l}\text { Santiago del } \\
\text { Estero }\end{array}$ & 2651 & 5318 & 93.5 & 0.9 & 5.6 & 253 & 25 & 1690 & 95 \\
\hline 55 & Jime & $\begin{array}{l}\text { Santiago del } \\
\text { Estero }\end{array}$ & 5786 & 5797 & 84.0 & 4.7 & 11.3 & 180 & 21 & 2308 & 261 \\
\hline 56 & Juan F. Ibarra & $\begin{array}{l}\text { Santiago del } \\
\text { Estero }\end{array}$ & 9263 & 6620 & 99.3 & 0.6 & 8.4 & 110 & 67 & 2121 & 179 \\
\hline 57 & Loreto & $\begin{array}{l}\text { Santiago del } \\
\text { Estero }\end{array}$ & 3151 & 5153 & 79.1 & 0.0 & 0.3 & 290 & 69 & 3495 & 9 \\
\hline 58 & Mitre & $\begin{array}{l}\text { Santiago del } \\
\text { Estero }\end{array}$ & 3384 & 7004 & 20.2 & 0.3 & 1.0 & 1725 & 19 & 1608 & 17 \\
\hline 59 & Moreno & $\begin{array}{l}\text { Santiago del } \\
\text { Estero }\end{array}$ & 16472 & 6543 & 78.2 & 5.5 & 16.3 & 282 & 224 & 1880 & 307 \\
\hline 60 & Ojo de Agua & $\begin{array}{l}\text { Santiago del } \\
\text { Estero }\end{array}$ & 6154 & 5834 & 44.6 & 3.2 & - & 574 & 65 & 0 & 0 \\
\hline
\end{tabular}




\begin{tabular}{|c|c|c|c|c|c|c|c|c|c|c|c|}
\hline \multirow[b]{2}{*}{ Código } & \multirow[b]{2}{*}{ Departamento } & \multirow[b]{2}{*}{ Provincia } & \multirow[b]{2}{*}{$\underset{\left(\mathrm{km}^{2}\right)}{\text { Superficie }}$} & \multirow[b]{2}{*}{$\begin{array}{l}\text { PPN áerea } \\
\left(\mathrm{Kg}_{\mathrm{MS}} \mathrm{ha}^{-}\right. \\
{ }^{1} \text {.año-1) }\end{array}$} & \multicolumn{3}{|c|}{ Cobertura (\%) } & \multicolumn{4}{|c|}{$\begin{array}{c}\text { Apropiación (Kg MS.ha- } \\
\left.{ }^{1} \cdot \text { año }^{-1}\right)\end{array}$} \\
\hline & & & & & $\begin{array}{l}\overrightarrow{\widetilde{J}} \\
\stackrel{\Xi}{\Xi} \\
\stackrel{\pi}{Z}\end{array}$ & 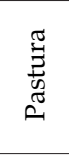 & 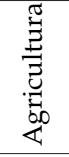 & 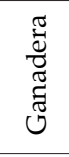 & $\begin{array}{l}\overrightarrow{\widetilde{J}} \\
\stackrel{0}{0} \\
\stackrel{0}{0} \\
\text { 足 }\end{array}$ & $\begin{array}{l}\frac{\pi}{8} \\
\stackrel{0}{\square} \\
\frac{0}{4}\end{array}$ & 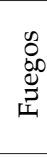 \\
\hline 61 & Pellegrini & $\begin{array}{l}\text { Santiago del } \\
\text { Estero }\end{array}$ & 6930 & 6406 & 92.4 & 0.8 & 6.8 & 146 & 140 & 2262 & 154 \\
\hline 62 & Quebrachos & $\begin{array}{l}\text { Santiago del } \\
\text { Estero }\end{array}$ & 3800 & 6557 & 98.6 & 1.4 & - & 399 & 14 & 0 & 0 \\
\hline 63 & Río Hondo & $\begin{array}{l}\text { Santiago del } \\
\text { Estero }\end{array}$ & 2056 & 5628 & 95.6 & 1.0 & 3.4 & 101 & 67 & 2120 & 73 \\
\hline 64 & Rivadavia & $\begin{array}{l}\text { Santiago del } \\
\text { Estero }\end{array}$ & 3664 & 7252 & 71.1 & 13.5 & 15.5 & 1241 & 1 & 2162 & 334 \\
\hline 65 & Robles & $\begin{array}{l}\text { Santiago del } \\
\text { Estero }\end{array}$ & 1312 & 5196 & 85.8 & 5.6 & 8.7 & 267 & 44 & 2184 & 189 \\
\hline 66 & Salavina & $\begin{array}{l}\text { Santiago del } \\
\text { Estero }\end{array}$ & 3380 & 6213 & 99.8 & 0.2 & - & 185 & 5 & 0 & 0 \\
\hline 67 & San Martín & $\begin{array}{l}\text { Santiago del } \\
\text { Estero }\end{array}$ & 2344 & 5337 & 98.3 & 0.2 & 1.5 & 104 & 91 & 1745 & 26 \\
\hline 68 & Sarmiento & $\begin{array}{l}\text { Santiago del } \\
\text { Estero }\end{array}$ & 1690 & 5793 & 99.4 & 0.2 & 0.4 & 108 & 243 & 926 & 4 \\
\hline 69 & Silípica & $\begin{array}{l}\text { Santiago del } \\
\text { Estero }\end{array}$ & 1003 & 5095 & 97.6 & 1.6 & 0.8 & 144 & 59 & 1828 & 15 \\
\hline
\end{tabular}

\section{ANEXO 3: ANALISIS DE COMPONENTES PRINCIPALES}

Para explorar el comportamiento integral del conjunto de variables e indicadores se realizó un análisis de componentes principales que consideró departamentos por variables indicadoras (Tabla 1) (INFOSTAT, Di Rienzo et al. 2009). Este análisis separó en primer lugar a departamentos con alta apropiación ganadera, mayor área dedicada a pasturas y agricultura y mayor densidad total de población de aquellos con predominancia de vegetación natural, altas tasas de desmonte actual, mayor presencia de ganado caprino, rodeos vacunos chicos, y poblaciones más pobres. En segundo lugar se diferenciaron departamentos con alta incidencia de fuegos, de clima más húmedo y ubicación más septentrional, con mayores tasas de apropiación forestal de la PPN y mayores niveles de pobreza (Figura A3).
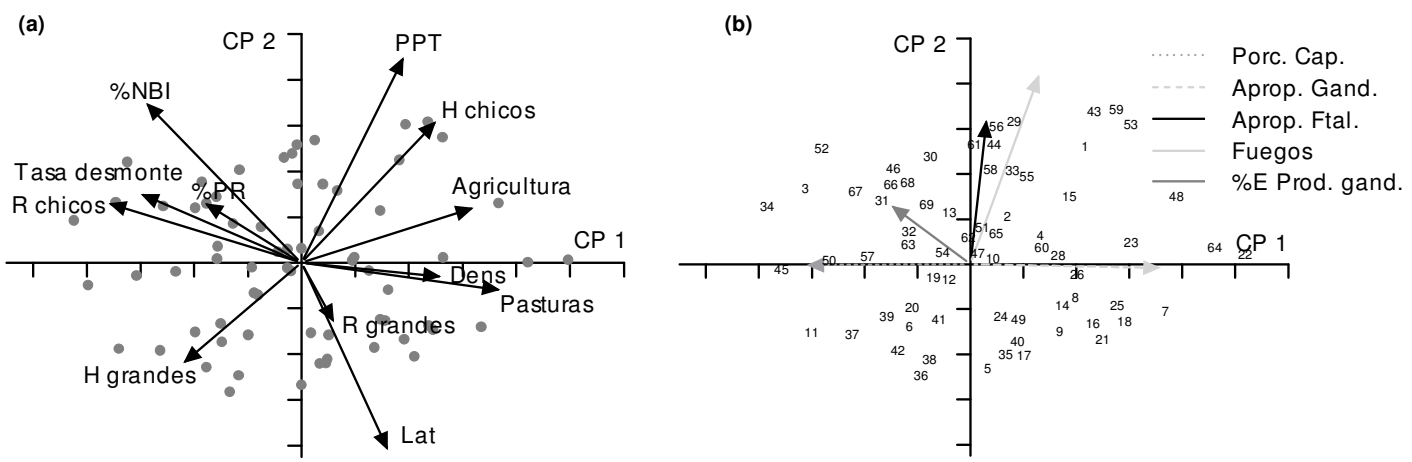

Figura A3. Análisis de componentes principales para el conjunto de variables socioeconómicas y climáticas e indicadores de apropiación sobre los 69 departamentos. Se representa en (a) los departamentos (puntos) y los vectores de variables socioeconómicas y climáticas y en (b) los departamentos (ver código en Anexo 2) y los vectores los indicadores de apropiación. El largo del vector indica el nivel de correlación con los ejes.

Figure A3. Principals components analysis for the set of socioeconomic and climatic variables and appropriation indicators of primary productivity of the 69 counties. Is depicted in (a) the counties (points) and the vectors of socioeconomic and climatic, and the (b) counties (see numbers in Appendix 2) and vectors appropriation indicators of primary productivity. The length of the vector indicates the level of correlation with the axes. 